\title{
Improved high-order adjacent vertex assignment sequence for similarity vertices and isomorphism identification of planar kinematic chains
}

\section{Liang Sun}

Zhejiang Sci-Tech University https://orcid.org/0000-0001-6476-477X

\section{Zhizheng Ye}

Zhejiang Sci-Tech University

Fuwei Lu

Zhejiang Sci-Tech University

Rongjiang Cui

Zhejiang Sci-Tech University

Chuanyu Wu ( $\nabla$ cywu@zstu.edu.cn )

Zhejiang Sci-Tech University

\section{Original Article}

Keywords: Similarity vertices, isomorphism identification, kinematic chains, topology, adjacency matrix

Posted Date: April 2nd, 2020

DOl: https://doi.org/10.21203/rs.3.rs-20134/v1

License: (c) (1) This work is licensed under a Creative Commons Attribution 4.0 International License. Read Full License 


\section{Title page}

Improved high-order adjacent vertex assignment sequence for similarity vertices and isomorphism identification of planar kinematic chains

\section{Liang Sun}

Faculty of Mechanical Engineering \& Automation, Zhejiang Sci-Tech University

928 Second Avenue, Xiasha Higher Education Zone, 310018 Hangzhou, China

E-mail: liangsun@zstu.edu.cn

\section{Zhizheng Ye}

Faculty of Mechanical Engineering \& Automation, Zhejiang Sci-Tech University 928 Second Avenue, Xiasha Higher Education Zone, 310018 Hangzhou, China

E-mail:201820501061@mails.zstu.edu.cn

\section{Fuwei Lu}

Faculty of Mechanical Engineering \& Automation, Zhejiang Sci-Tech University 928 Second Avenue, Xiasha Higher Education Zone, 310018 Hangzhou, China

E-mail: 2017g506015@mais.zstu.edu.cn

\section{Rongjiang Cui}

Faculty of Mechanical Engineering \& Automation, Zhejiang Sci-Tech University 928 Second Avenue, Xiasha Higher Education Zone, 310018 Hangzhou, China

E-mail: cuirongjiang2009@163.com

\section{Chuanyu Wu}

Faculty of Mechanical Engineering \& Automation, Zhejiang Sci-Tech University 928 Second Avenue, Xiasha Higher Education Zone, 310018 Hangzhou, China

E-mail: cywu@zstu.edu.cn

\section{Corresponding author: Chuanyu Wu E-mail: cywu@zstu.edu.cn}




\title{
Improved high-order adjacent vertex assignment sequence for similarity vertices and isomorphism identification of planar kinematic chains
}

\author{
Liang Sun1 • Zhizheng Ye1 $・$ Fuwei Lu1 $・$ Rongjiang Cui1 $・$ Chuanyu Wu 1
}

\begin{abstract}
Isomorphism identification is fundamental to synthesis and innovative design of kinematic chains (KCs). The identification can be performed accurately by using the similarity of KCs. However, there are very few researches on isomorphism identification based on the properties of similarity vertices. In this paper, an improved high-order adjacent vertex assignment (IHAVS) sequence method is proposed to seek out the similarity vertices and identify the isomorphism of the planar KCs. First, the specific definition of IHAVS is described. Through the calculation of the IHAVS, the adjacent point value sequence reflecting the uniqueness of the structural features is established. Based on the value sequence, all possible similarity vertices, corresponding relations and isomorphism discrimination can be realized. By checking the topological diagrams of $\mathrm{KCs}$ of different number of links, the correctness of the proposed method are verified. Finally, the method is used to find the similarity vertices of all the 9-link 2-DOF(degree of freedom) planar KCs.
\end{abstract}

Keywords: Similarity vertices $•$ isomorphism identification • kinematic chains $\bullet$ topology $\bullet$ adjacency matrix

\section{Introduction}

At present, the multi-component and multi-freedom mechanism is widely used in various mechanical equipment, involving engineering machinery, medical machinery, agricultural machinery, etc. The innovative design of mechanism structure is still the key step of product upgrading [1-2]. The correct solution of the similarity vertices [3] can not only reduce the number of isomorphism identification, but also provide a theoretical basis for the selection of rack, input and output components. In 1992, Yan [4] proposed a new mechanism design technology, called regenerative KCs method, which essentially represents the logical reasoning of the regeneration process of KCs. In logic reasoning, the similarity vertices should be analyzed to improve the efficiency of innovative design and reduce redundant design scheme. In the same year, Hwang [5] divided the similarity into symmetry similarity, transfer similarity, row similarity and irregular similarity, and proposed the relationship code method of weighted line graph to distinguish the similarity vertices of functional components. Although Hwang's method is simple, it has the situation of misjudgment.

In mechanism synthesis, isomorphism identification is fundamental. Many researches have been carried out and a large number of methods and theories have been developed. Kong et al. [6] first applied the artificial neural network technology to isomorphism identification, and established the neural network model of topological graph. Chang et al. [7] proposed to identify $\mathrm{KCs}$ isomorphism by comparing the eigenvalues and eigenvectors of the corresponding adjacency matrix of topological graphs for the first time; Cubillo and Wan [8] found that the original theory had errors, proposed the necessary and sufficient conditions for eigenvalues and eigenvectors of adjacent matrices of isomorphic KCs to modified the methods; Later, Sunkari and Schmidt [9] found that this method has certain limitations. This method fails when the number of components in the KCs is greater than 14. Xiao et al. [10] creatively combined ant colony algorithm and artificial immune algorithm to identify the isomorphism of KCs. Ding and Huang [11-14] standardized topological graph according to certain rules, used the normal adjacency matrix to identify the $\mathrm{KCs}$ isomorphism, and developed the algorithm of related isomorphism identification. Galán-Marín et al. [15] first used the multi value neural network method to identify the isomorphism of KCs. In the same year, Dargar et al. [16] proposed to identify isomorphism by comparing the first-order and second-order adjacent component values of the $\mathrm{KCs}$, but 
this method has the defect that it can't describe the uniqueness of each vertex in the topological graph. Yang et al. [17] proposed a method of detecting isomorphism based on the correlation matrix of KCs. By using this method, the sufficiency of isomorphism identification was obtained on the basis of vertex correspondence. Zeng et al. [18] proposed a partition matching algorithm to the identify isomorphism. Yang et al. [19] proposed a hybrid immune algorithm by combining immune algorithm, genetic algorithm and local search algorithm, and realized the recognition of isomorphism by using this algorithm. Shang et al. [20] proposed to use the method of optimized circuit simulation to identify the KCs isomorphism. In 2018, Sun et al. [21] proposed to use joint matrix to uniquely describe the structure of $\mathrm{KCs}$, obtain the corresponding component and joint information from joint matrix, and use joint matrix, component and joint to identify isomorphism. In the same year, Rai and Punjabi [22] first used connection number and entropy to ignore tolerance gap to identify isomorphism. He et al. [23] used the dynamic IHAVS to find the similarity vertices and distinguish the isomorphism of the topological graph. However, when using its IHAVS to find the similar points, there are counter examples, such as Figure 3 and the topological diagram with the serial number of 9 in the appendix.

Although all the above synthesis methods can identify the isomorphism, they can't accurately find the similarity vertices that are beneficial for mechanism selection and isomorphism identification. In order to solve this problem, a method is proposed to find the similarity vertices and identify the isomorphism of the $\mathrm{KCs}$ based on the improved IHAVS sequence. And the correctness of this method is verified.

The rest of this paper is organized as follows: Section 2 introduces the basic knowledge of graph theory. In Section 3 , the initial value rule and the definition of IHAVS of topological graph are described. Several examples are given to find the similarity vertices of KCs. In Section 4, based on the correct solution of the similarity vertices, the concrete steps of identifying the isomorphism of the KCs are given. Section 5, conclusion.

\section{Basic concepts}

\subsection{Topological model of KCs}

KCs can be described by an undirected topological graph in which the vertices and edges represent the components and joints of the KCs respectively; The link relationship between vertices can be expressed by adjacency matrix $A=\left[a_{i j}\right]_{n \times n}$, where $\mathrm{n}$ is the total number of vertices in the topological graph, and the adjacency matrix is a real symmetric matrix. The value rules of each element are as follows:

$$
A=\left[a_{i j}\right]_{n \times n}== \begin{cases}1 & \text { if vertex } i \text { is adjacent to vertex } j \\ 0 & \text { otherwise (including } i=j \text { ) }\end{cases}
$$

The degree of vertex $\mathrm{i}$ in topological graph is defined as the number of edges connected with vertex $i$; it can also be determined by the sum of i-th column (row) of adjacency matrix A. As shown in Figure 1, the topological diagram of the 9-link 2-DOF $\mathrm{KC}$ is expressed as (b), and the corresponding adjacency matrix is A. Vertices 2, 4, 6, 7 and 9 are two-dimensional points, while vertices $1,3,5$ and 8 are three-dimensional points.

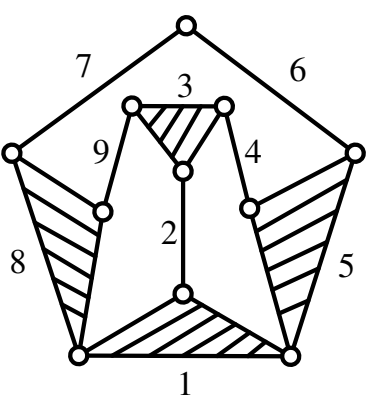

(a)

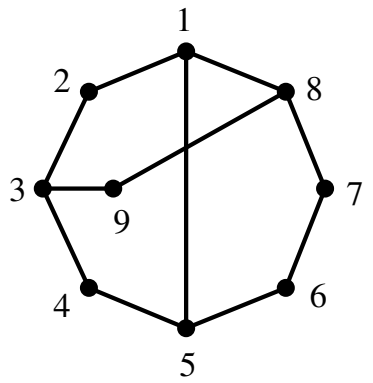

(b)
Figure 1 2-DOF KCs (a) and its corresponding topological diagram (b)

$$
\mathbf{A}=\left[\begin{array}{lllllllll}
0 & 1 & 0 & 0 & 1 & 0 & 0 & 1 & 0 \\
1 & 0 & 1 & 0 & 0 & 0 & 0 & 0 & 0 \\
0 & 1 & 0 & 1 & 0 & 0 & 0 & 0 & 1 \\
0 & 0 & 1 & 0 & 1 & 0 & 0 & 0 & 0 \\
1 & 0 & 0 & 1 & 0 & 1 & 0 & 0 & 0 \\
0 & 0 & 0 & 0 & 1 & 0 & 1 & 0 & 0 \\
0 & 0 & 0 & 0 & 0 & 1 & 0 & 1 & 0 \\
1 & 0 & 0 & 0 & 0 & 0 & 1 & 0 & 1 \\
0 & 0 & 1 & 0 & 0 & 0 & 0 & 1 & 0
\end{array}\right]
$$

\subsection{Definition of similarity vertices}

We consider a graph as labeled when its vertices are labeled by the integers $1,2, \ldots, n$. In this regard, a labeled graph is mapped into another labeled graph when the $n$ integers are permuted. For some permutations, a labeled graph may map into itself. A set of those permutations, which map the graph into itself, form a group that is a 
group of automorphisms. It is also said to be a vertex-induced group. Two vertices of a graph contained in the same permutation cycle of a vertex-induced group of automorphisms are deemed similar.

As shown in Figure 1 (b), based on the component similarity classification proposed in reference [5], vertices 4 and 9,5 and 8,6 and 7 are symmetrically similar, the possible number of automorphism groups is $2 ! \times 2 ! \times 2=8$, and listed as follows:

$$
\begin{aligned}
& p_{1}=[1][2][3][4][9][5][8][6][7] \\
& p_{2}=[1][2][3][4 / 9][5][8][6][7] \\
& p_{3}=[1][2][3][4][9][5 / 8][6][7] \\
& p_{4}=[1][2][3][4][9][5][8][6 / 7], \\
& p_{5}=[1][2][3][4 / 9][5 / 8][6][7] \\
& p_{6}=[1][2][3][4 / 9][5][8][6 / 7] \\
& p_{7}=[1][2][3][4][9][5 / 8][6 / 7] \\
& p_{8}=[1][2][3][4 / 9][5 / 8][6 / 7]
\end{aligned}
$$

where $p_{1}$ and $p_{8}$ can transform the adjacency matrix into itself. The chain automorphism group is $\left\{p_{1}, p_{8}\right\}$. Vertices 4 and 9, 5 and 8,6 and 7 are in the automorphism cycle $p_{8}$ and are thus similar.

\subsection{Isomorphism of $\mathrm{KCs}$}

If one-to-one mapping $f$ exists for the two graphs $G_{1}=\left(V_{1}, E_{1}\right)$ and $G_{2}=\left(V_{2}, E_{2}\right)$, and the following expression is satisfied:

$$
u, v \in V_{1},[u, v] \in E_{1} \Leftrightarrow[f(u), f(v)] \in E_{2},
$$

then graphs G1 and G2 are isomorphic. As shown in Figure 2, $G(a)$ and $G(b)$ are isomorphic.
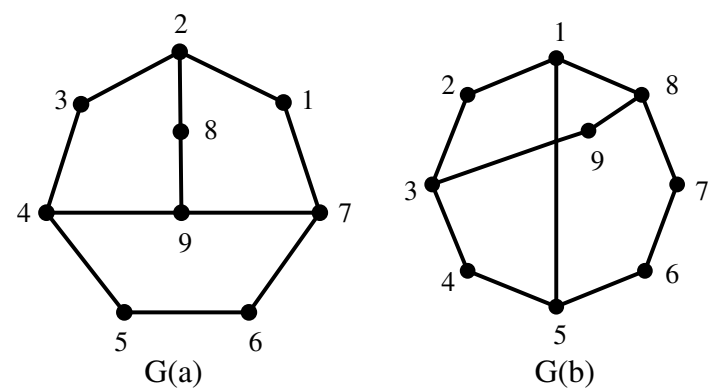

Figure 2 Isomorphic topological graph

\section{IHAVS sequence and solution of similarity vertices}

\subsection{Initial value and IHAVS}

For any given topological graph, the number of $3 \mathrm{j}$ of Fibonacci sequence [24-25] is assigned according to the size of each vertex degree, where $j$ is a positive integer. According to the vertex degrees, the values of $j$ are $1,5,21$, $89 . .$. The vertices with equal degree are assigned the same value. This assignment method is defined as the initial value, and the initial value sequence is recorded as $S^{0}$. As shown in Figure 1 (b), since vertices 2, 4, 6, 7 and 9 are two-dimensional points, the initial value of vertices $2,4,6$, 7 and 9 is 1 . Vertices $1,3,5$ and 8 are three-dimensional points, the initial value of vertices $1,3,5$ and 8 is 5 , that is, the initial value sequence is $S^{0}=\{5 、 1 、 5 、 1 、 5 、 1 、 1$ 、 5、 1$\}$.

Note that the $r$-th order adjacent vertex value (AVV) of vertex $i$ in topological graph $\mathrm{G}$ is $S_{i}^{r}$ :

$$
s_{i}^{r}=s_{i}^{r-1}+\frac{1}{10} \sum_{j=1}^{n} s_{j}^{r-1} a_{i, j} \sum_{k=1}^{n} a_{k, j},
$$

where $n$ is the total number of vertices in the topology. $a_{i, j}$ is the element corresponding to row $i$ and column $j$ of adjacency matrix A. $S_{i}^{r-1}$ is the $(r-1)$-th order AVV of vertex $i$. It can be seen from the analysis that the larger $r$ is, the more fully $r$-order AVV characterizes the vertex of topological graph. And the larger $r$ is, the more complex its calculation is. So it is of great significance to choose the right $r$. In this paper, $r$ is taken as the integral part of $\sqrt{n}+d$, where $\mathrm{d}$ changes dynamically according to the specific situation.

The weight of the weighting part is $1 / 10$ instead of $1 / n$. The advantage of this value is that the decimal point of the AVV of order $r$ is exactly equal to $r$, which can avoid rounding error.

The sequence of the $r$-order AVV of all vertices is $S^{r}=\left\{s_{1}^{r}, s_{2}^{r}, s_{3}^{r}, \ldots \ldots, s_{n}^{r}\right\}$. The $r$-th order AVV of vertex $i$ is determined by the initial value of vertex $i$, degree of vertex $i$, the degree of the vertex adjacent to vertex $i$ and the (r-1)-th order AVV of the vertex adjacent to vertex $i$. Hence, the $r$-th order AVV can uniquely describe the characteristics of each vertex in the topological graph. That is, in the $r$-th order AVV sequence $S^{r}$, if the $r$-th order AVV of the two vertices are the same, the two vertices are similar, otherwise they are not similar. The solution of similarity vertices is of great significance to the selection of frame, input and output components. The correct solution of similarity vertices can reduce the number of isomorphism identification and improve the overall efficiency of mechanism synthesis. 


\subsection{Examples of finding similarity vertices}

Example 1: As shown in Figure 3, the corresponding topological diagram of the 11-link 2-DOF KCs (a) is shown in Figure (b). The process of finding the similarity vertices by using the IHAVS before and after correction is as follows:

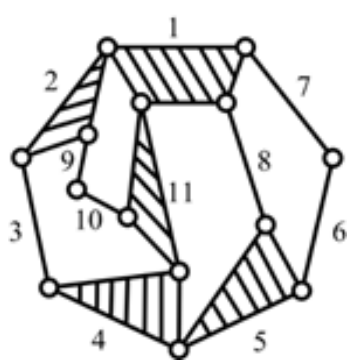

(a)

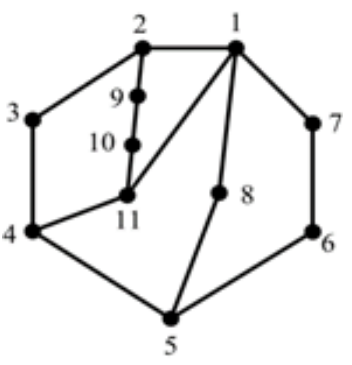

(b)
Figure 3 11-link 2-DOF KCs (a) and its topological diagram (b)

IHAVS before modification [23]: The value of $r$ before correction is 4 , the initial value of the prime number sequence according to the vertex degree of the topological graph is recorded as $S^{0}$, and the fourth order AVV sequence is recorded as $S_{4}$, the solution results before correction are shown in Table 1.

Table 1 The initial value and fourth order AVV sequence before modification of Figure 3

\begin{tabular}{|c|c|}
\hline Sequence name & Result \\
\hline Prime column initial value & $\mathrm{S}_{0}=\{3 、 2 、 1 、 2 、 2 、 1 、 1 、 1 、 1 、 1 、 1 、$ \\
\hline $\begin{array}{c}\text { Fourth order AVV sequence } \\
\text { before modification }\end{array}$ & $\begin{array}{c}\mathrm{S}_{4}=\{6.7982 、 4.9415 、 3.3155 、 4.9982 \\
4.5172 、 2.7740 、 3.2588 、 3.7398 、 2.7740 \\
2.8345 、 5.4225\}\end{array}$ \\
\hline
\end{tabular}

According to the fourth order AVV sequence $S_{4}$, $s_{6}^{4}=s_{9}^{4}=2.7740$, vertices 6 and 9 are similar. But in fact, there is no similarity vertices in this topological diagram. Considering whether the value of $r$ is too small, which leads to the correctness of the conclusion, we have solved the similarity of the fifth order, eighth order and even eleventh order AVV series. The conclusion is still that 6 and 9 are similar, so there are problems in the formula before modification.

Improved IHAVS sequence: total vertices of topology $n=11, \quad r_{0}=\sqrt{n}+1 \approx 4, r_{1}=\sqrt{n}+2 \approx 5$. The initial value of vertex degree in topological graph is $S_{0}$. By using the initial value, the fourth and fifth order AVV sequence obtained from equation (3) is recorded as $S_{4}$ and $S_{5}$, the specific solution results after correction are shown in Table 2.

Table 2 The modified initial value and $\mathrm{r}$, $\mathrm{r} 1$-th order AVV sequence of Figure 3

\begin{tabular}{cc}
\hline Sequence name & Result \\
\hline Fibonacci initial value & $S_{0}=\{21 、 5 、 1 、 5 、 5 、 1 、 1 、 1 、 1 、 1 、 5\}$ \\
Revised ro-th order & $S_{4}=\{104.68740 、 75.804400 、 44.669800 、$ \\
AVV sequence & $55.733800 、 42.657800 、 28.308400 、 63.574400 、$ \\
& $72.624800 、 32.934000 、 35.573400 、 86.00300\}$ \\
Revised r1-th order & $S_{5}=\{180.46946 、 133.20012 、 84.13126 、$ \\
AVV sequence & $103.26600 、 79.56458 、 53.82062 、 111.11104 、$ \\
& $127.29710 、 62.79000 、 67.96110 、 151.71278\}$ \\
\hline
\end{tabular}

According to the fourth and fifth order AVV sequence $S_{4}$ and $S_{5}$, the adjacency values of each vertex are different, so there are no similar vertices in the topological graph. Therefore, in the innovative design of the mechanism, there are 11 different ways to choose the frame.

Example 2: As shown in Figure 4, there is a topology with 12 vertices and 15 edges. The solution process of the similarity vertices is as follows:

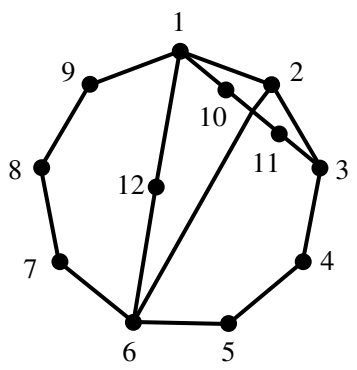

Figure 4 A topology with 12 vertices and 15 edges

Total vertices of topology $n=12, \quad r_{0}=\sqrt{n}+1 \approx 4$, $r_{1}=\sqrt{n}+2 \approx 5$. The initial value of vertex degree in topological graph is $S_{0}$; By using the initial value, the fourth and fifth order AVV sequence obtained from equation (3) is recorded as $S_{4}$ and $S_{5}$, the specific solution results after correction are shown in Table 3:

Table 3 The initial value and ro, r1-th order AVV sequence of Figure 4

\begin{tabular}{cc}
\hline Sequence name & Result \\
\hline Initial value & $\mathrm{S}_{0}=\{21 、 5 、 5 、 1 、 1 、 21 、 1 、 1 、 1 、 1 、$ \\
Revised ro-th order AVV & $\mathrm{S}_{4}=\{116.53090 、 140.05410 、 60.32370 、$ \\
sequence & $31.44010 、 64.96580 、 116.53090 、 63.98200 、$ \\
& $26.95360 、 63.98200 、 64.96580 、 31.44010 、$ \\
& $118.75280\}$ \\
\hline
\end{tabular}


Revised $\mathrm{r}_{1}$-th order AVV $\quad \mathrm{S}_{5}=\{208.08725 、 251.37593 、 114.91597$, sequence

62.53037、117.86618、208.08725、

115.98508、52.54640、115.98508、

$117.86618 、 62.53037 、 211.97752\}$

According to the fourth order AVV sequence $S_{4}$,

$$
\begin{gathered}
s_{1}^{4}=s_{6}^{4}=116.53090, \\
s_{4}^{4}=s_{11}^{4}=31.44010, \\
s_{5}^{4}=s_{10}^{4}=64.96580, \\
s_{7}^{4}=s_{9}^{4}=63.98200 ;
\end{gathered}
$$

According to the fifth order AVV sequence $S_{5}$,

$$
\begin{gathered}
s_{1}^{5}=s_{6}^{5}=208.08725, \\
s_{4}^{5}=s_{11}^{5}=62.53037, \\
s_{5}^{5}=s_{10}^{5}=117.86618, \\
s_{7}^{5}=s_{9}^{5}=115.98508 .
\end{gathered}
$$

Vertices 1 and 6, 4 and 11, 5 and 10, 7 and 9 are similar to each other; Vertices 2, 3, 8 and 12 have different adjacency values with other points, so they have no similarity vertices. Therefore, in the innovative design of the mechanism, there are 8 different ways to choose the frame.

Example 3: As shown in Figure 5, there is a topology with 21 vertices and 29 edges. The solution process of the similarity vertices is as follows:

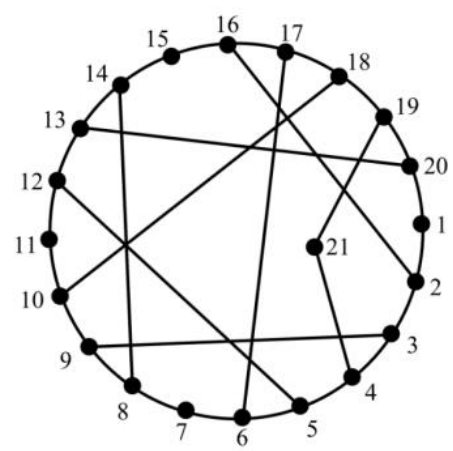

Figure 5 A topology with 21 vertices and 29 edges

Total vertices of topology $n=21, \quad r_{0}=\sqrt{n}+1 \approx 5$, $r_{1}=\sqrt{n}+2 \approx 6$. The initial value of vertex degree in topological graph is $S_{0}$. By using the initial value, the fifth and sixth order AVV sequence obtained from equation (3) is recorded as $S_{5}$ and $S_{6}$, the specific solution results after correction are shown in Table 4:

Table 4 The initial value and ro, r1-th order AVV sequence of
Figure 5

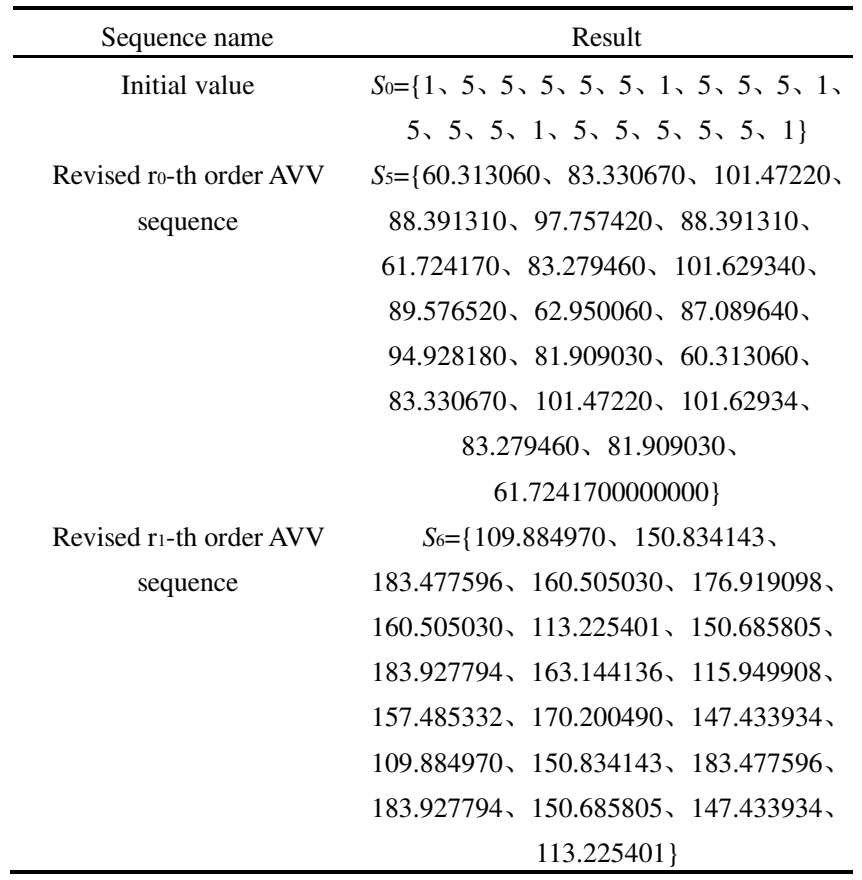

According to the fifth order AVV sequence $S_{5}$,

$$
\begin{gathered}
s_{1}^{5}=s_{15}^{5}=60.313060, \\
s_{2}^{5}=s_{16}^{5}=83.330670, \\
s_{3}^{5}=s_{17}^{5}=101.47220, \\
s_{4}^{5}=s_{6}^{5}=88.391310, \\
s_{7}^{5}=s_{21}^{5}=61.724170, \\
s_{8}^{5}=s_{19}^{5}=83.279460, \\
s_{9}^{5}=s_{18}^{5}=101.629340, \\
s_{14}^{5}=s_{20}^{5}=81.909030 ;
\end{gathered}
$$

According to the sixth order AVV sequence $S_{6}$,

$$
\begin{gathered}
s_{1}^{6}=s_{15}^{6}=109.884970, \\
s_{2}^{6}=s_{16}^{6}=150.834143, \\
s_{3}^{6}=s_{17}^{6}=183.477596, \\
s_{4}^{6}=s_{6}^{6}=160.505030, \\
s_{7}^{6}=s_{21}^{6}=113.225401, \\
s_{8}^{6}=s_{19}^{6}=150.685805, \\
s_{9}^{6}=s_{18}^{6}=183.927794, \\
s_{14}^{6}=s_{20}^{6}=147.433934 .
\end{gathered}
$$

Vertices 1 and 15, 2 and 16, 3 and 17, 4 and 6, 7 and 21, 8 and 19, 9 and 18, 14 and 20 are similar to each other; Vertices 5, 10, 11, 12, and 13 have different adjacency values with other points, so they have no similarity vertices. 
Therefore, in the innovative design of the mechanism, there are 13 different ways to choose the frame.

In the end of this paper, we use the IHAVS sequence to find the similarity vertices of all the 9-link 2-DOF topological diagrams. See the appendix for the specific solution results, which further verifies the correctness of this method.

\section{Steps and examples of isomorphic identification}

\subsection{Initial value and IHAVS}

In this paper, if the values of two sequences are the same and the numbers of the same values are the same, then the two sequences correspond to each other, and the corresponding relationship is recorded as ${ }_{a}^{b} M$. Such as sequences $S_{a}=\{1 、 3 、 2 、 4 、 5\}$ and $S_{b}=\{3 、 4 、 1 、 5 、 2\}$, this two sequences correspond to each other and their corresponding relations ${ }_{a}^{b} M=\{3 、 1 、 5 、 2 、 4\}$. The first element 3 of the corresponding relation indicates that the first element of the sequence $S_{a}$ corresponds to the third element of the sequence $S_{b}$, the second element 1 of the corresponding relation indicates that the second element of the sequence $S_{a}$ corresponds to the first element of the sequence $S_{b}$, and so on.

For any given two topological graphs, if they are isomorphic, their corresponding vertex degrees must be the same, and the vertex degrees at the same distance from the two corresponding vertices must be the same. It can be seen from equation (3) that the $r$-th order AVV of vertex $i$ is determined by the initial value of vertex $i$, degree of vertex $i$, the degree of the vertex adjacent to vertex $i$ and the $(r-1)$-th order AVV of the vertex adjacent to vertex $i$. Therefore, the corresponding relationship can be found by the $r$-order AVV sequence $S_{a}^{r}$ and $S_{b}^{r}$. The adjacency matrix of topological graph $\mathrm{G}(\mathrm{a})$ is transformed first by row and then by column by corresponding relationship. Then, the adjacency matrix after transformation is compared with the adjacency matrix of topological graph $\mathrm{G}(\mathrm{b})$. If they are the same, they are isomorphic. Otherwise, they are non-isomorphic. The specific steps of isomorphism identification are as follows:

Step1: For any given two topological graphs G(a) and $\mathrm{G}(\mathrm{b})$, the initial values of each vertex degree are recorded as $S_{a}^{0}$ and $S_{b}^{0}$. The $r$-order AVV sequences $S_{a}^{r}$ and $S_{b}^{r}$ are calculated by equation (3) to distinguish the similarity vertices between $\mathrm{G}(\mathrm{a})$ and $\mathrm{G}(\mathrm{b})$. If the number of groups of two topological graphs is different or the number of groups is the same but the number of similar points of corresponding groups is different, the two topological graphs are non-isomorphic. Otherwise, go to step 2. If there are no similarity vertices between the two topologies and the sequence $S_{a}^{r}$ and $S_{b}^{r}$ has no corresponding relationship, the two topologies are non-isomorphic. Otherwise, there is a set of corresponding relationship ${ }_{a}^{b} M$, go to step 5.

Step2: The indefinite value of sequence $S_{a}^{r}$ is assigned according to the following rules: Select the vertex with few similar points and the smallest label as $i$, change its initial value to the sum of the original initial value and the $(p+1)$ Fibonacci number, where $p$ is the number of indefinite assignment. For example, the vertex selected for the first initial value is $j$, its corresponding adjacent point value is recorded as $t$, the new initial value of vertex $j$ is recorded as $g_{1}$, and its new $r$-order AVV sequence calculated by equation (3) is $S_{a, 1}^{r}$, If there are still duplicate values in $S_{a, 1}^{r}$, continue to assign indefinite values to the duplicate values until the sequence $S_{a, p}^{r}$ without duplicate values is obtained, and two sets of substitution sets $\mathrm{T}=\left(t 1, t_{2}, \ldots, t_{p}\right)$ and $\mathrm{G}=\left(g_{1}, g_{2}, \ldots, g_{p}\right)$ are obtained.

Step3: According to $\mathrm{T}=\left(t_{1}, t_{2}, \ldots, t_{p}\right)$ and $\mathrm{G}=\left(g_{1}, g_{2}, \ldots, g_{p}\right)$ to assign a fixed value to sequence $S_{b}^{r}$, the specific rules are as follows: Obtain the same vertex as the first element $t_{1}$ in the replacement set $\mathrm{T}$ in the sequence $S_{b}^{r}$, and assign a new initial value to this vertex as the first element $g_{1}$ in the replacement set G, the new r-order AVV sequence $S_{b, 1}^{r}$ calculated by equation (3) according to the new initial value; Similarly, if the $r$-order AVV in the new sequence $S_{b, 1}^{r}$ is equal to $t_{2}$, the new initial value given to the vertex is $g_{2}$, and the new $r$-order AVV sequence calculated from equation (3) according to the new initial value is $S_{b, 2}^{r}$; If there are multiple values in $S_{b, 1}^{r}$ which are the same as $t_{2}$, multiple new $r$-order AVV sequences $S_{b, 2}^{r}$ can be obtained. If no value in the sequence $S_{b, k-1}^{r}$ is equal to $t_{k}$ $(k \leq p)$ in the $k$-th fixed value assignment process, the fixed value assignment fails, those two topologies are non-isomorphic, and the program terminates. Otherwise, one or more sets of final sequence $S_{b, p, q}^{r}$ can be obtained, where $q$ is the number of sets of final sequence.

Step4: Compare the sequence $S_{a, p}^{r}$ and $S_{b, p, q}^{r}$. If the sequence $S_{b, p, q}^{r}$ doesn't correspond to the sequence $S_{a, p}^{r}$, the two topologies are non-isomorphic and the program is terminated. Otherwise, there are one or more sets of sequence $S_{b, p, q}^{r}$ corresponding to sequence $S_{a, p}^{r}$, and one or more sets of correspondence ${ }_{a}^{b} M$ can be obtained.

Step5: According to the corresponding relation, the adjacency matrix $A$ corresponding to the topological graph G(a) is transformed first by row and then by column, one or more transformed adjacency matrix $A_{2}$ is obtained. 
Compared $A_{2}$ with the adjacency matrix $B$ corresponding to the topological graph $\mathrm{G}(\mathrm{b})$, if there is no corresponding, the two topological graphs are non-isomorphic and the program terminates; otherwise, the two topological graphs are isomorphic.

\subsection{Examples of isomorphic identification}

Example 1: As shown in Figure 6, there are 11-link 2-DOF topological diagrams $\mathrm{G}(\mathrm{a})$ and $\mathrm{G}(\mathrm{b})$ and its adjacency matrices are $\mathrm{A}$ and $\mathrm{B}$ respectively. The isomorphism identification process is as follows:

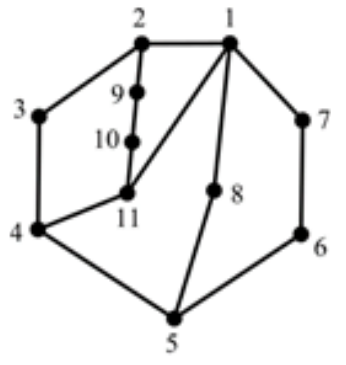

G(a)

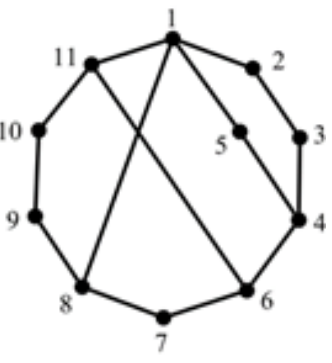

$\mathrm{G}(\mathrm{b})$
Figure 6 A topology with 11-link and 2-DOF

Step1: In G(a), vertices 3, 6, 7, 8, 9 and 10 are two-dimensional points, vertices $2,4,5$ and 11 are three-dimensional points, vertex 1 is four-dimensional point, and the initial value sequence of each vertex is $S_{a}^{0}$ . In the same way, for $G(b)$, according to the degree of each vertex, the initial value sequence of each vertex is recorded as $S_{b}^{0}$. On the basis of the initial value, the $r$-order adjacency sequence $S_{a}^{r}$ and $S_{b}^{r}$ are obtained from equation (3). The process of determining $r$ value is the same as that of distinguishing similarity vertices, $r=\sqrt{n}+1+1 \approx 5$. The initial value and the solution results of the fifth order AVV sequence are shown in Table 5.

Table 5 The initial value and fifth order adjacent point value sequence of $\mathrm{G}(\mathrm{a})$ and $\mathrm{G}(\mathrm{b})$

\begin{tabular}{|c|c|}
\hline Sequence name & Result \\
\hline Initial value of G(a) & $S_{a}^{0}=\{21 、 5 、 1 、 5 、 5 、 1 、 1 、 1 、 1 、 1 、$ \\
\hline Initial value of $G(b)$ & $S_{b}^{0}=\{21 、 1 、 1 、 5 、 1 、 5 、 1 、 5 、 1 、 1 、$ \\
\hline The fifth order AVV sequence & $\begin{array}{c}S_{a}^{5}=\{180.46946 、 133.20012 、 84.13126 、 \\
103.26600 、 79.56458 、\end{array}$ \\
\hline of $\mathrm{G}(\mathrm{a})$ & $\begin{array}{r}53.82062 、 111.11104 、 127.29710 、 \\
62.79000 、 67.96110 、 151.71278\}\end{array}$ \\
\hline $\begin{array}{l}\text { The fifth order AVV sequence } \\
\text { of } G(b)\end{array}$ & $\begin{array}{c}S_{b}^{5}=\{180.46946 、 111.11104 、 53.82062 、 \\
79.56458 、 127.29710 、\end{array}$ \\
\hline
\end{tabular}

103.26600、84.13126、133.20012、

62.79000、67.96110、151.71278\}

It can be seen from the sequence $S_{a}^{5}$ and $S_{b}^{5}$ that there is no similarity between $\mathrm{G}(\mathrm{a})$ and $\mathrm{G}(\mathrm{b})$, but there is a corresponding relationship between the sequence $S_{a}^{5}$ and $S_{b}^{5}$. The corresponding relationship ${ }_{a}^{b} M=\{1,8 、 7 、 6$ 、 4、3、2、5、9、10、11\}.

Step5: On the basis of ${ }_{a}^{b} M$, the adjacency matrix A corresponding to the topological graph $\mathrm{G}(\mathrm{a})$ is transformed, first by row and then by column, into an adjacency matrix A2. Compared A2 with the adjacency matrix B corresponding to the topological graph $\mathrm{G}(\mathrm{b})$, they are the same, so the two topological graphs are isomorphic.

Example 2: As shown in Figure 7, G(a) and $\mathrm{G}(\mathrm{b})$ are topological diagrams with 30 vertices and 40 edges. Isomorphism identification process is as follows:

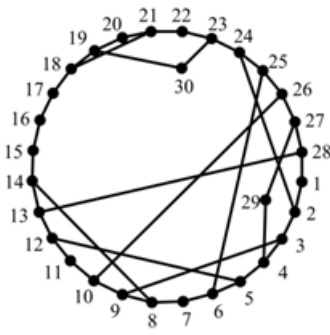

$\mathrm{G}(\mathrm{a})$

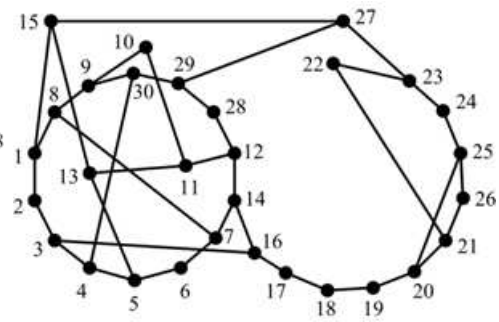

$\mathrm{G}(\mathrm{b})$
Figure 7 Topological diagrams with 30 vertices and 40 edges

Step1: In G(a), vertices 1, 7, 11, 15, 16, 17, 20, 22, 29 and 30 are two-dimensional points, the rest vertices are three-dimensional points, and the initial value sequence of each vertex is $S_{a}^{0}$. Similarly, the initial value sequence of topological graph $\mathrm{G}(\mathrm{b})$ is recorded as $S_{b}^{0}$; On the basis of the initial value, the $r$-order adjacency sequence $S_{a}^{r}$ and $S_{b}^{r} \quad$ are obtained from equation (3), where $r=\sqrt{n}+1+1 \approx 7$. The initial value and the solution results of the seventh order AVV sequence are shown in Table 6.

Table 6 The initial value and fifth order adjacent point value sequence of $G(a)$ and $G(b)$

\begin{tabular}{|c|c|}
\hline Sequence name & Result \\
\hline $\begin{array}{l}\text { Initial value of } \\
\qquad G(a)\end{array}$ & $\begin{array}{c}S_{a}^{0}=\{1,5,5,5,5,5,1,5,5,5,1,5,5 、 \\
5,1,1 、 1 、 5 、 5 、 1 、 5 、 1 、 5 、 5 、 5 、 5 、 5 、 \\
5,1,1\}\end{array}$ \\
\hline $\begin{array}{l}\text { Initial value of } \\
\text { G(b) }\end{array}$ & $\begin{array}{c}S_{b}^{0}=\{5,1,5,5,5,1,5,5,5,1,5,5,5 、 \\
5,5,5,1 、 1 、 1 、 5 、 5 、 1 、 5 、 1 、 5 、 1 、 5 、 \\
1,5,5\}\end{array}$ \\
\hline e seven & $S_{a}^{7}=\{204.9462274 、 290.6737873 、 337.96$ \\
\hline
\end{tabular}




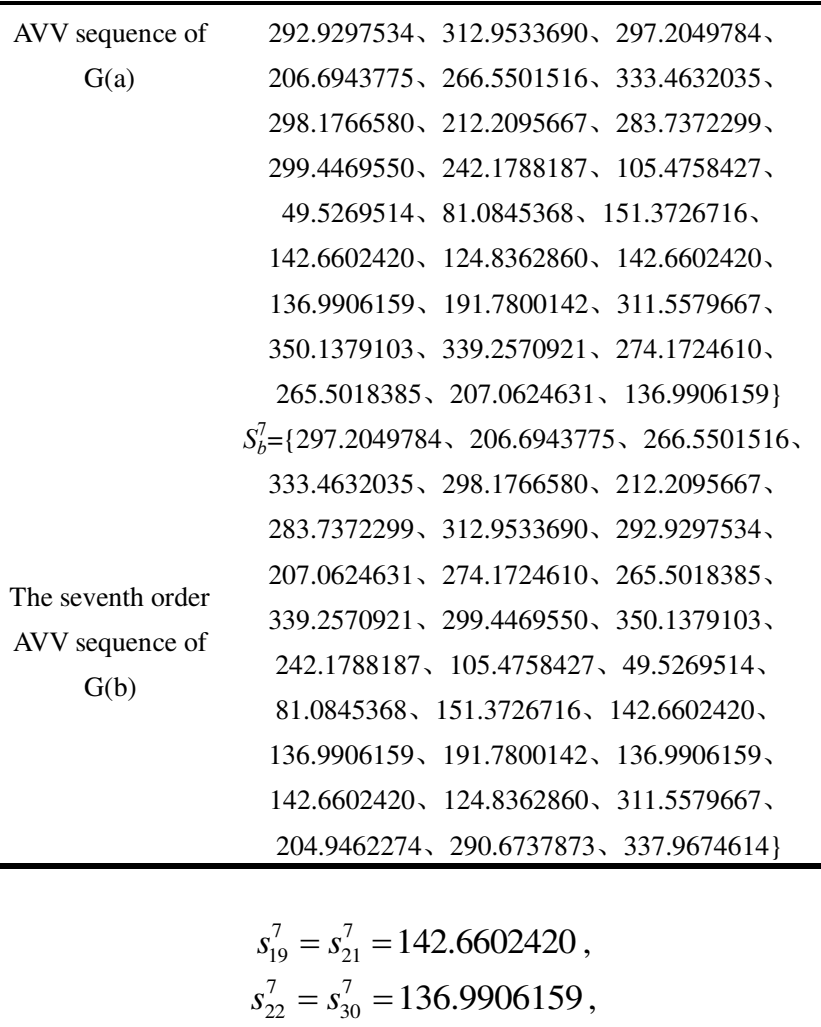

so in $\mathrm{G}(\mathrm{a})$, vertices 19 and 21,22 and 30 are similar to each other;

$$
\begin{gathered}
s_{21}^{7}=s_{25}^{7}=142.6602420, \\
s_{22}^{7}=s_{24}^{7}=136.9906159,
\end{gathered}
$$

so in $\mathrm{G}(\mathrm{b})$, vertices 21 and 25, 22 and 34 are similar to each other. Since the number of groups of similar points is the same as the number of vertices of corresponding groups, go to step 2 .

Step2: Assign indefinite value to sequence $S_{a}^{7}$. Since the number of two groups of similar points is the same, the minimum vertex label is vertex 19, and its AVV is 142.6602420. According to the rule of indefinite value assignment, the initial value of vertex 19 is changed to 6 , so $S_{a}^{0}=\{1 、 5 、 5 、 5 、 5 、 5 、 1 、 5 、 5 、 5 、 1 、 5 、 5 、 5$, $1 、 1 、 1 、 5 、 6 、 1 、 5 、 1 、 5 、 5 、 5 、 5 、 5 、 5 、 1 、 1\}$. Its corresponding fifth order adjacency value $S_{a, 1}^{7}$ $=\{204.9818026 、 290.8968073 、 338.0032310 、 292.9333012$ 、 321.9569168、297.2407480、206.6978443、266.5519066、 $333.4668161 、 298.1802058 、 212.2097125 、 283.7374405$ 、 $299.4485642 、 242.1944715 、 105.6159547 、 50.3498682$ 、 $84.5465128 、 159.0086076 、 150.9347260 、 131.6648284$ 、 $148.5472140 、 139.8790777 、 194.3560842 、 312.4795343$ 、 $350.3620643 、 339.2929103 、 274.1759602 、 265.5043171$ 、 $207.0627547 、 142.6318225\}$ 。 It can be seen that there is no duplicate value in sequence $S_{a, 1}^{7}$, its final sequence is $S_{a, 1}^{7}$, the two substitution sets are $\mathrm{T}=(142.6602420)$ and $\mathrm{G}=(6)$ 。

Step3: According to $\mathrm{T}=(142.6602420)$ and $\mathrm{G}=(6)$ to assign a fixed value to sequence $S_{b}^{7}$. Since the values of the $21_{\mathrm{st}}$ and 25 th bits in sequence $S_{b}^{7}$ are equal to 142.6602420, assign values to these two positions respectively to obtain the seventh order AVV sequence $S_{b, 1, q}^{7}$, where $q=1$ represents the adjacency value sequence obtained by changing the initial value of vertex 21 in topological graph $\mathrm{G}(\mathrm{b}), q=2$ represents the adjacency value sequence obtained by changing the initial value of vertex 25 in topological graph $\mathrm{G}(\mathrm{b})$, and the final sequence result is shown in Table 7.

Table 7 Seven order AVV sequence after G(b) fixed value

\begin{tabular}{|c|c|}
\hline$q$ & $S_{b, 1, q}^{7}$ \\
\hline$q=1$ & $\begin{array}{c}S_{b, 1,1}^{7}=\{297.2407480 、 206.6978443 、 266.5519066 、 \\
\text { 333.4668161、298.1802058、212.2097125、 } \\
\text { 283.7374405、321.9569168、292.9333012、 } \\
\text { 207.0627547、274.1759602、265.5043171、 } \\
\text { 339.2929103、299.4485642、350.3620643、 } \\
\text { 242.1944715、105.6159547、50.3498682、 } \\
\text { 84.5465128、159.0086076、150.9347260、 } \\
\text { 142.6318225、194.3560842、139.8790777、 } \\
148.5472140 、 131.6648284 、 312.4795343 、 \\
204.9818026 、 290.8968073 、 338.0032310\}\end{array}$ \\
\hline$q=2$ & $\begin{array}{c}S_{b, 1,2}^{7}=\{297.2407480 、 206.6978443 、 266.5519066 、 \\
\text { 333.4668161、298.1802058、212.2097125、 } \\
\text { 283.7374405、321.9569168、292.9333012、 } \\
\text { 207.0627547、274.1759602、265.5043171、 } \\
\text { 339.2929103、299.4485642、350.3620643、 } \\
\text { 242.1944715、105.6159547、50.3498682、 } \\
\text { 84.5465128、159.0086076、148.5472140、 } \\
\text { 139.8790777、194.3560842、142.6318225、 } \\
\text { 150.9347260、131.6648284、312.4795343、 } \\
204.9818026 、 290.8968073 、 338.0032310\}\end{array}$ \\
\hline
\end{tabular}
assignment

Step4: Comparing $S_{a, 1}^{7}$ and $S_{b, 1, q}^{7}$ can get two corresponding relations: ${ }_{a}^{b} M_{1}=\{28 、 29 、 30 、 9 、 8 、 1 、 2 、$ $3 、 4 、 5 、 6 、 7 、 14 、 16 、 17 、 18 、 19 、 20 、 21 、 26 、$ $25 、 24 、 23 、 27 、 15 、 13 、 11 、 12 、 10 、 22\},{ }_{a}^{b} M_{2}=\{28 、$ $29 、 30 、 9 、 8 、 1 、 2 、 3 、 4 、 5 、 6 、 7 、 14,16 、 17$. $18 、 19 、 20 、 25 、 26 、 21 、 22 、 23 、 27 、 15 、 13 、 11 、$ 12、10、24\}.

Step5: On the basis of ${ }_{a}^{b} M$, the adjacency matrix A corresponding to the topological graph $\mathrm{G}(\mathrm{a})$ is transformed first by row and then by column, adjacency matrix A2 is obtained. Compared A2 with the adjacency matrix B corresponding to the topological graph $\mathrm{G}(\mathrm{b})$,they are the 
same, so the two topological graphs are isomorphic.

Example 3: As shown in Figure 8, G(a) and G(b) are topological diagrams with 15 vertices and 27 edges. Isomorphism identification process is as follows:

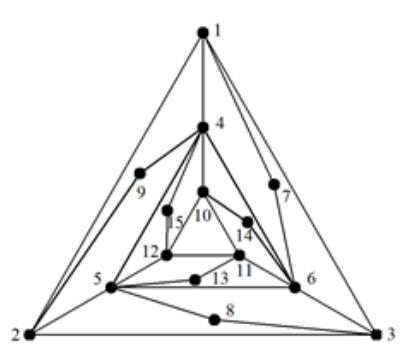

G(a)

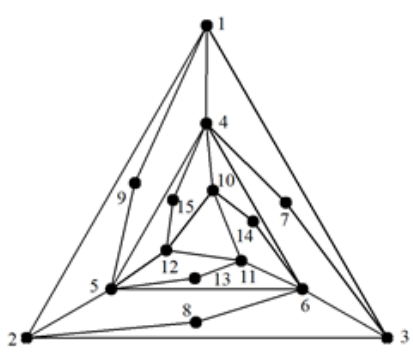

G(b)
Figure 8 Topological diagrams with 15 vertices and 27 edges

Step1: In G(a), vertices 7, 8, 9, 13, 14 and 15 are two-dimensional points, vertices $1,2,3,10,11$ and 12 are four-dimensional points, vertices 4,5 and 6 are six-dimensional points, the initial value sequence of each vertex is $S_{a}^{0}$. Similarly, the initial value sequence of topological graph $\mathrm{G}(\mathrm{b})$ is recorded as $S_{b}^{0}$; On the basis of the initial value, the $r$-order adjacency sequence $S_{a}^{r}$ and $S_{b}^{r}$ are obtained from equation (3), where $r=\sqrt{n}+1+1 \approx 5$. The initial value and the solution results of the seventh order AVV sequence are shown in Table 8.

Table 8 The initial value and fifth order adjacent point value sequence of $G(a)$ and $G(b)$

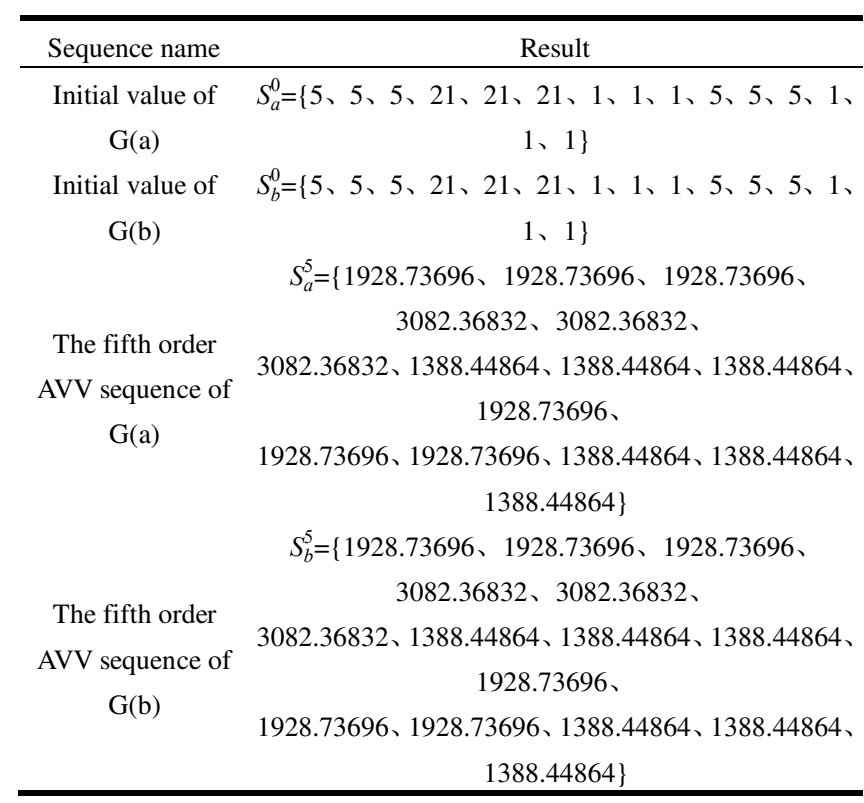

$$
\begin{aligned}
s_{1}^{5}= & s_{2}^{5}=s_{3}^{5}=s_{10}^{5}=s_{11}^{5}=s_{12}^{5}=1928.73696, \\
& s_{4}^{5}=s_{5}^{5}=s_{6}^{5}=3082.36832, \\
s_{7}^{5}= & s_{8}^{5}=s_{9}^{5}=s_{13}^{5}=s_{14}^{5}=s_{15}^{5}=1388.44864,
\end{aligned}
$$

so in G(a), vertices $1,2,3,10,11$ and 12 are similar to each other, vertices 4,5 and 6 are similar to each other, and vertices 7, 8, 9, 13, 14 and 15 are similar to each other. Similarly, in $\mathrm{G}(\mathrm{b})$, vertices $1,2,3,10,11$ and 12 are similar to each other, vertices 4,5 and 6 are similar to each other, and vertices 7, 8, 9, 13, 14 and 15 are similar to each other. Since the number of groups of similar points is the same as the number of vertices of corresponding groups, go to step 2.

Step2: Assign indefinite value to sequence $S_{a}^{5}$. Since the number of two groups of similar points is the same, the minimum vertex label is vertex 4 , change vertex 4 to 22 , so $S_{a, 1}^{5}=\{1954.70976 、 1953.51456 、 1952.82720 、 3124.25280$ 、 $3122.07104 、 3122.07104 、 1406.45056 、 1405.07584$ 、 $1408.33312 、 1954.70976 、 1952.82720 、 1953.51456$ 、 1405.07584、1406.45056、1408.33312\}. There are still duplicate values in the sequence $S_{a, 1}^{5}$, and the sequence $S_{a, 2}^{5}$ can be obtained by assigning a new value 7 to component $1, \quad S_{a, 2}^{5}=\{1985.87200 、 1982.35040$ 、 $1981.66304 、 3158.88320 、 3154.19136 、 3155.10784$ 、 $1426.36352 、 1421.22688 、 1427.23360 、 1970.65728$ 、 $1967.31552 、 1968.00288 、 1415.58976 、 1418.46208$ 、 $1421.59648\}$. It can be seen that there is no duplicate value in sequence $S_{a, 2}^{5}$, its final sequence is $S_{a, 2}^{5}$, the two substitution sets are $\mathrm{T}=(3082.36832 、 1954.70976)$ and $\mathrm{G}=(22 、 7)$ 。

step3: According to $\mathrm{T}=(3082.36832 、 1954.70976)$ and $\mathrm{G}=(22 、 7)$ to assign a fixed value to sequence $S_{b}^{5}$. Since the values of the $24 \mathrm{th}, 25 \mathrm{th}$, and $26 \mathrm{th}$ bits in sequence $S_{b}^{7}$ are equal to $3082.36832,6$ groups of final data were obtained, as follows:

$S_{b-1}^{5}=\{1985.87200 、 1981.66304 、 1982.35040$ 、 $3158.88320 、 3155.10784 、 3154.19136 、 1427.23360$ 、 $1421.22688 、 1426.36352 、 1970.58816 、 1966.88544$ 、 1968.50208、1416.08896、1417.82464、1421.73472\};

$S_{b-2}^{5}=\{1970.58816 、 1966.88544 、 1968.50208$ 、 $3158.88320 、 3154.19136 、 3155.10784 、 1421.73472$ 、 $1416.08896 、 1417.82464 、 1985.87200 、 1981.66304 、$ $1982.35040 、 1421.22688 、 1426.36352 、 1427.23360\}$;

$S_{b-3}^{5}=\{1982.38040 、 1985.87200 、 1981.66304$ 、 $3154.19136 、 3158.88320 、 3155.10784 、 1421.22688$ 、 $1426.36352 、 1427.23360 、 1966.88544 、 1968.50208$ 、 $1970.58816 、 1421.73472 、 1416.08896 、 1417.82464\}$;

$S_{b-4}^{5}=\{1968.50208 、 1970.58816 、 1966.88544$ 、 $3155.10784 、 3158.88320 、 3154.19136 、 1416.08896$ 、 $1417.82464 、 1421.73472 、 1981.66304 、 1982.35040 、$ 
1985.87200、1427.23360、1421.22688、1426.36352\};

$S_{b-5}^{5}=\{1981.66304 、 1982.35040 、 1985.87200$ 、

$3155.10784 、 3154.19136 、 3158.88320 、 1426.63520$ 、

$1427.23360 、 1421.22688 、 1968.50208 、 1970.58816$ 、

$1966.88544 、 1417.82464 、 1421.73472 、 1416.08896\}$;

$S_{b-6}^{5}=\{1966.88544 、 1968.50208 、 1970.58816$ 、

$3154.19136 、 3155.10784 、 3158.88320 、 1417.82464$ 、

$1421.73472 、 1416.088996 、 1982.35040 、 1985.87200 、$

1981.66304、1426.36352、1427.23360、1421.22688\};

Step4: Because none of the six final sequences can correspond to the sequence $S_{a, 2}^{5}$, the two topologies are non-isomorphic.

\section{Conclusions}

In this paper, an improved IHAVS sequence method is proposed not only to find similarity vertices to improve the efficiency of isomorphism identification, but also to provide a theoretical basis for selecting the functional parts of the mechanism. The IHAVS sequence is employed to describe the uniqueness of each vertex in topological graph. Further, with the IHAVS sequence, the similarity vertices can be found accurately, and the reliability of isomorphic discrimination can be guaranteed by confirming the similarities. In order to prove the correctness of this method, the topological diagrams of 9, 11, 21 and 30 links $\mathrm{KCs}$ have been checked. Moreover, the similarity library of 9-link 2-DOF KCs is given for the first time. Our method improves the overall efficiency of mechanism synthesis.

\section{Declaration}

\section{Acknowledgements}

The first author sincerely acknowledges the CSC scholarship for his study and visiting abroad.

\section{Funding}

This work was supported in part by the National Natural Science Foundation of China (Grant Nos. 51975534), in part by Zhejiang Provincial Natural Science Foundation of China (Grant No. LY19E050021), in part by the 151 Talent Plan of Zhejiang Province, and in part by the Project of Zhejiang Provincial Young and Middle-aged Discipline Leaders. Any opinions, findings, conclusions, or recommendations expressed in this publication are those of the authors and do not necessarily reflect the views of the National Science Foundation of China, Zhejiang Province, and Zhejiang Sci-Tech University.

\section{Availability of data and materials}

The datasets supporting the conclusions of this article are included within the article.

\section{Authors' contributions}

The author' contributions are as follows: Liang Sun in charge of the deducing of the formula and the entire process of the study; Zhizheng Ye compiled the most important part of the program; Fuwei Lu wrote the manuscript; Rongjiang Cui assisted with examples verification and data analyses; Chuanyu $\mathrm{Wu}$ focused on the structure of the manuscript.

\section{Competing interests}

The authors declare no competing financial interests.

\section{Consent for publication \\ Not applicable}

\section{Ethics approval and consent to participate Not applicable}

\section{References}

[1] Wang J Y, Zhao J S, Chu F L, et al. Innovative design of the lifting mechanisms for forklift trucks[J]. Mechanism and Machine Theory, 2010, 45(12): 1892-1896.

[2] Hsieh W H, Chen S J. Innovative design of cam-controlled planetary gear trains[J]. International Journal of Engineering and Technology Innovation, 2011, 1(1): 1.

[3] Sun L, Cui R, Ye Z, et al. Similarity recognition and isomorphism identification of planar kinematic chains[J]. Mechanism and Machine Theory, 2020, 145: 103678.

[4] H.S. Yan, A methodology for creative mechanism design, Mechanism and machine theory, 27 (1992) 235-242.

[5] W.-M. Hwang, Y.-W. Hwang, Computer-aided structural synthesis of planar kinematic chains with simple joints, Mechanism and Machine Theory, 27 (1992) 189-199.

[6] Kong F G, Li Q, Zhang W J. An artificial neural network approach to mechanism kinematic chain isomorphism identification[J]. Mechanism and Machine Theory, 1999, 34(2): 271-283.

[7] Chang Z, Zhang C, Yang Y, et al. A new method to mechanism kinematic chain isomorphism identification[J]. Mechanism and Machine Theory, 2002, 37(4): 411-417.

[8] Cubillo J P, Wan J. Comments on mechanism kinematic chain isomorphism identification using adjacent matrices[J]. Mechanism and Machine Theory, 2005, 40(2): 131-139.

[9] Sunkari R P, Schmidt L C. Reliability and efficiency of the existing spectral methods for isomorphism detection[J]. Journal of Mechanical Design, 2006, 128(6): 1246-1252.

[10] Renbin Xiao, Tao Z, Liu Y. Isomorphism identification of kinematic chains using novel evolutionary approaches[J]. Journal of Computing and Information Science in Engineering, 2005, 5(1): 18-24.

[11] Ding H , Huang Z . A unique representation of the kinematic chain and the atlas database[J]. Mechanism \& Machine Theory, 2007, 42(6):637-651.

[12] Ding H F, Huang Z. The establishment of the canonical perimeter 
topological graph of kinematic chains and isomorphism identification[J]. Journal of mechanical design, 2007, 129(9): 915-923.

[13] Ding H, Huang Z, Mu D . Computer-aided structure decomposition theory of kinematic chains and its applications[J]. Mechanism \& Machine Theory, 2008, 43(12):1596-1609.

[14] Ding H F, Huang Z. Isomorphism identification of graphs: Especially for the graphs of kinematic chains[J]. Mechanism and Machine Theory, 2009, 44(1): 122-139.

[15] Galán-Marín G, López-Rodríguez D, Mérida-Casermeiro E. A new multivalued neural network for isomorphism identification of kinematic chains $[\mathrm{J}]$. Journal of Computing and Information Science in Engineering, 2010, 10(1): 1-4.

[16] Dargar A, Khan R A, Hasan A. Application of link adjacency values to detect isomorphism among kinematic chains[J]. International Journal of Mechanics and Materials in Design, 2010, 6(2): 157-162.

[17] Yang F, Deng Z, Tao J, et al. A new method for isomorphism identification in topological graphs using incident matrices[J]. Mechanism and Machine theory, 2012, 49: 298-307.

[18] Zeng K, Fan X, Dong M, et al. A fast algorithm for kinematic chain isomorphism identification based on dividing and matching vertices[J]. Mechanism and Machine Theory, 2014, 72: 25-38.

[19] Yang P, Zeng K, Li C, et al. An improved hybrid immune algorithm for mechanism kinematic chain isomorphism identification in intelligent design[J]. Soft Computing, 2015, 19(1): 217-223.

[20] Shang H, Gao X, Shi R, et al. A kinematic chain isomorphism identification algorithm using optimized circuit simulation method[C]. 2016 35th Chinese Control Conference (CCC). IEEE, 2016: 4061-4067.

[21] Sun W, Kong J, Sun L. A joint-joint matrix representation of planar kinematic chains with multiple joints and isomorphism identification[J]. Advances in Mechanical Engineering, 2018, 10(6): $1-10$.

[22] Rai R K, Punjabi S. Kinematic chains isomorphism identification using link connectivity number and entropy neglecting tolerance and clearance[J]. Mechanism and Machine Theory, 2018, 123: 40-65.

[23] He L, Liu F, Sun L, et al. Isomorphic identification for kinematic chains using variable high-order adjacency link values[J]. Journal of Mechanical Science and Technology, 2019, 33(10): 4899-4907.

[24] Lewis H. The Fibonacci Sequence and Poetry[J]. Math Horizons, 2018, 26(1): 2-2.

[25] Ghosh N. Fibonacci numbers in real life applications[J]. Mugberia Gangadhar Mahavidyalaya, 2018, 1: 62-69.

\section{Biographical notes}

Liang Sun, born in 1981, is an Associate Professor at Faculty of Mechanical Engineering \& Automation, Zhejiang Sci-Tech University, Hangzhou, China. He received his doctoral degree in Mechanical Engineering from Zhejiang Sci-Tech University in 2014. His researches focus on mechanical design and optimization

Tel: +86-571-86843756; E-mail: liangsun@zstu.edu.cn.

Zhizheng Ye, born in 1996, is currently a master candidate at Faculty of Mechanical Engineering \& Automation, Zhejiang Sci-Tech University, China.

E-mail:201820501061@mails.zstu.edu.cn

Fuwei Lu, born in 1990, is currently a master candidate at Faculty of Mechanical Engineering \& Automation, Zhejiang
Sci-Tech University, China.

E-mail: 2017g506015@mais.zstu.edu.cn

Rongjiang Cui, born in 1983, is currently a Ph.D. candidate at Faculty of Mechanical Engineering \& Automation, Zhejiang Sci-Tech University, China. His research interest is mechanism synthesis.

E-mail: 1004108862@qq.com

Chuanyu Wu, born in 1976, is an Professor at Faculty of Mechanical Engineering \& Automation, Zhejiang Sci-Tech University, Hangzhou, China. He received his doctoral degree in Mechanical Engineering from Zhejiang University in 2002. His researches focus on mechanical design and optimization.

E-mail: cywu@zstu.edu.cn

\section{Appendix}

The solution results of the similarity vertices of 9-link 2-DOF topological graph

\begin{tabular}{|c|c|c|c|}
\hline Number & Topological graph & 5th order AVV & $\begin{array}{c}\text { Similarity } \\
\text { vertices }\end{array}$ \\
\hline 01 & & $\begin{array}{c}S_{5}=\{170.46720 、 \\
112.06664 、 76.47140 、 \\
135.63688 、 99.13144 、 \\
132.26808 、 56.77396 、 \\
37.45448 、 104.36936\}\end{array}$ & $\begin{array}{c}\text { No } \\
\text { similarity } \\
\text { vertices }\end{array}$ \\
\hline 02 & & $\begin{array}{c}S_{5}=\{71.08127 、 \\
52.01055 、 45.37576 、 \\
56.26729 、 45.37576 、 \\
52.01055 、 26.19135 、 \\
14.17132 、 26.19135\}\end{array}$ & $\begin{array}{l}2 、 6 \\
\text { 3、 } 5 \\
\text { 7、 } 9\end{array}$ \\
\hline 03 & & $\begin{array}{c}S_{5}=\{63.25629 、 \\
47.83189 、 48.97344 、 \\
63.25629 、 47.83189 、 \\
48.97344 、 25.24023 、 \\
13.96180 、 25.24023\}\end{array}$ & $\begin{array}{l}1 、 4 \\
2 、 5 \\
3 、 6 \\
7 、 9\end{array}$ \\
\hline 04 & & $\begin{array}{c}\mathrm{S}_{5}=\{140.21483 、 \\
78.24833 、 104.60506 、 \\
150.95043 、 121.02453 、 \\
93.77489 、 39.07613 、 \\
32.72850 、 95.88112\}\end{array}$ & $\begin{array}{c}\text { No } \\
\text { similarity } \\
\text { vertices }\end{array}$ \\
\hline 05 & & $\begin{array}{c}S_{5}=\{66.28000 、 \\
35.71896 、 35.71896 、 \\
66.28000 、 65.61200 、 \\
65.61200 、 31.20216 、 \\
15.80896 、 31.20216\}\end{array}$ & $\begin{array}{l}1,4 \\
2 、 3 \\
5,6 \\
7,9\end{array}$ \\
\hline 06 & & $\begin{array}{c}S_{5}=\{137.23511 、 \\
61.39755 、 44.95635 、 \\
94.15589 、 120.95741 、 \\
150.00271 、 98.65220 、 \\
49.43344 、 98.65220\}\end{array}$ & 7、 9 \\
\hline
\end{tabular}


07

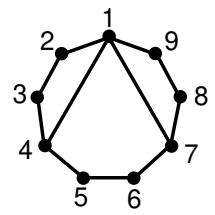

08

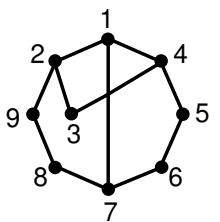

09

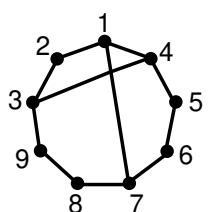

10
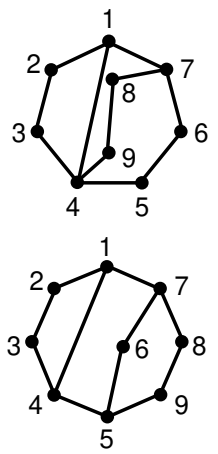

12

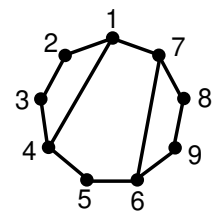

13

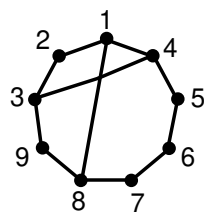

14
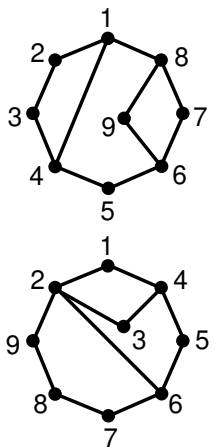

$\mathrm{S}_{5}=\{168.92496$ 、

52.58049、77.51145、

33.99564、33.99564、

15
111.71880、74.96248、

127.63248、60.72576、 60.72576、127.63248

74.96248、111.71880

$S_{5}=\{69.41399 、$

52.29035、44.53510、

52.29035、29.86593、

29.05491、48.54633、

$29.05491 、 29.86593\}$

$S_{5}=\{61.42065$ 、

47.54689、48.65018、

59.40811、33.23670、

28.69359、44.86968、

$27.89427 、 28.70763\}$

$\mathrm{S}_{5}=\{134.48647$ 、

77.29689、103.35258、

146.19009、98.76702、

52.58049、98.76702\}

$\mathrm{S}_{5}=\{58.59085$ 、

58.59085、47.65520、

42.19042、47.65520、

$28.65555 、 28.65555\}$

$\mathrm{S}_{5}=\{58.51321$ 、

33.19164、29.45955、

47.72816、42.19978、

47.72816、58.51321、

$33.19164 、 29.45955\}$

$\mathrm{S}_{5}=\{62.36139$ 、

48.55435、52.34464、

59.83457、29.77632、

14.75956、25.19439、

48.04986、43.24762\}

$\mathrm{S}_{5}=\{54.13288$ 、

32.05296、29.06130、

45.73652、36.89758、

34.20284、37.65774、

48.45064、37.65574\}

$\mathrm{S}_{5}=\{113.63052$ 、

2、 9

3、 8

4、 7

5、 6

2、 4

5、 9

6、 8

\section{No}

similarity

vertices

5、9

6、 8

$1 、 4$

2、 3

5、 7

8、 9

1、 7

2、 8

3、 9

4、 6

No

similarity

vertices

7、 9

23

46.46800、113.63052、

75.23528、72.31564、

118.46264、54.03544、 $35.98048 、 95.35032\}$

19

$1 、 3$

24

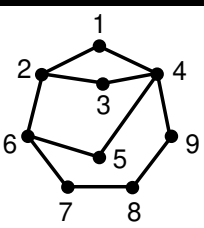

$\mathrm{S}_{5}=\{108.67353 、$

84.04417、108.67353、

124.44466、104.16141、

$1 、 3$

68.05285、32.11337、

$30.62094 、 85.68844$ \}

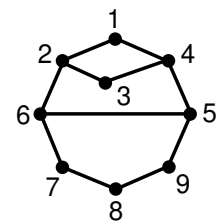

$\mathrm{S}_{5}=\{43.97710 、$

51.49246、43.97710、

51.49246、58.86359、

58.86359、29.72814、

15.55732、29.72814\}

1、3

2、 4

5、 6

7、 9

$\mathrm{S}_{5}=\{69.15460 、$

120.58448、69.15460、
61.28672、108.76700、

143.19352、96.85136、

49.12288、96.85136\}

$S_{5}=\{112.16572 、$

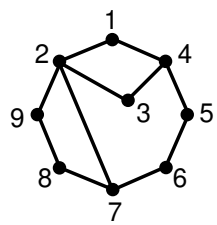

46.89024、112.16572、

67.53256、35.91852、

$1 、 3$

54.24024、118.08136、

71.73680、102.79816\}

$\mathrm{S}_{5}=\{106.88745$ 、

79.47405、106.88745、1、3

121.00068、88.03814、 5、9

44.49701、53.12997、

6、 8

$44.49701 、 88.03814\}$

$$
\mathrm{S}_{5}=\{41.17126 \text { 、 }
$$

45.21793、41.17126、

45.21793、42.32141、

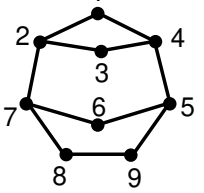

39.63154、42.32141、

$27.25263 、 27.25263\}$

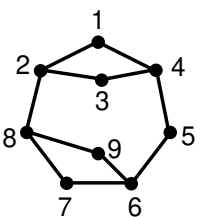

$S_{5}=\{36.41113$ 、

44.24617、36.41113、

33.13100、31.90264、

33.13100、36.41113、

$44.24617 、 36.41113\}$

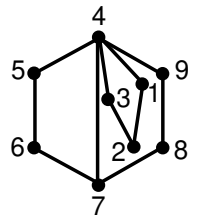

$\mathrm{S}_{5}=\{143.60163$ 、

64.62876、143.60163、1、3

$207.93361 、 149.03957 、 \quad 5 、 9$

91.73027、169.87119、

6、 8

91.73027、149.03957\}

$\mathrm{S}_{5}=\{106.88384$ 、

51.87104、106.88384、 $1 、 3$

169.68040、132.85640、

5、 7

98.80936、132.85640、

8、 9

3

9

5、 7

8、9

1、3、7、

2、 8

4、 6

3
9
8

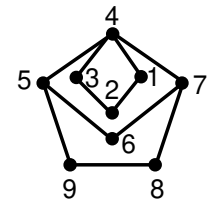


25

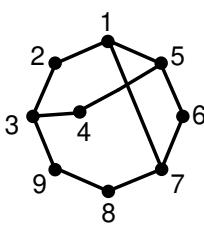

26

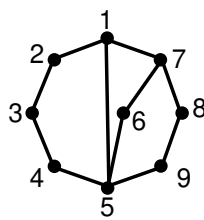

27

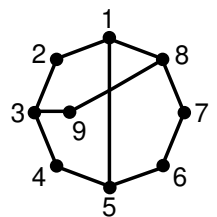

28

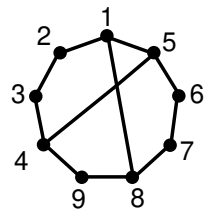

29

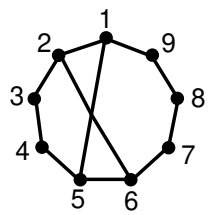

30
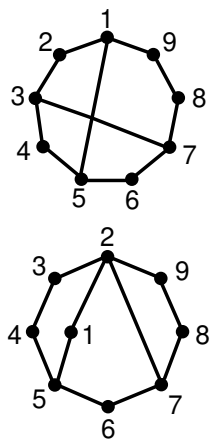

32

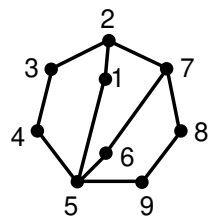

$\mathrm{S}_{5}=\{57.22116$ 、

40.52020、31.21248、

37.10710、50.01376、

42.84148、47.29652、

$27.68154 、 22.78296\}$

$\mathrm{S}_{5}=\{137.32339$ 、

59.69297、36.88806、

96.25120、150.08907、

121.39773、96.60729、

57.63149、100.38646]

$\mathrm{S}_{5}=\{56.28744$ 、

41.27836、34.84160、

37.10476、46.33568、

28.46916、28.46916、

$46.33568 、 37.10476\}$

$\mathrm{S}_{5}=\{58.51321$ 、

33.19164、29.45955、

47.72816、58.51321、

33.19164、29.45955、

$47.72816 、 42.19978\}$

$\mathrm{S}_{5}=\{65.68808$ 、

66.20392、35.71428、

35.71428、66.20392、

65.68808、31.20684、

15.80896、31.20684

$\mathrm{S}_{5}=\{42.39281$ 、

40.40140、45.14653、

41.16190、45.14653、

40.40140、42.39281、

$27.25731 、 27.25731\}$

$\mathrm{S}_{5}=\{107.74252$ 、

143.70056、97.08824、

45.48460、56.02920、

67.68532、120.29512、

72.28960、101.89664]

$\mathrm{S}_{5}=\{102.74853$ 、

66.30201、48.57689、

88.40510、120.14868、

102.74853、66.30201、

48.57689、88.40510\}
No

similarity

vertices

No

similarity

vertices

4、 9

5、 8

6. 7

$1 、 5$

2、 6

3、 7

4、 8

1、 6

2、 5

3、 4

7、 9

1、 7

2、 6

3、 5

8、 9

No

similarity

vertices

1、 6

2、 7

3、 8

4、 9
33
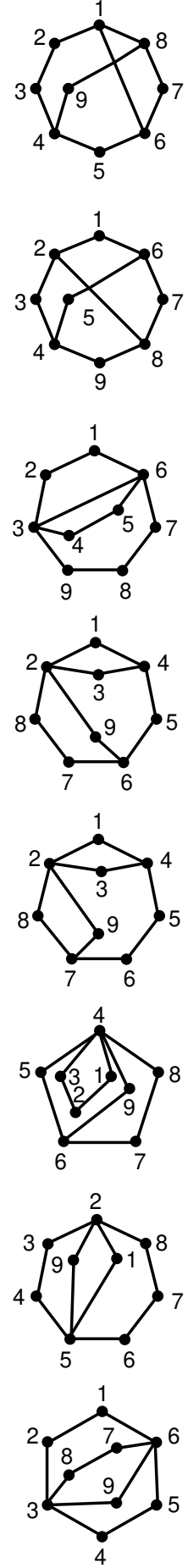

$S_{5}=\{55.06504 、$

31.26300、23.37276、 $30.57528 、 36.89992$ 、 49.41304、43.39480、

5、9

6、 8

49.41304、36.89992

$$
\mathrm{S}_{5}=\{36.41113 、
$$

44.24617、36.41113、

$33.13100 、 31.90264$ 、

33.13100、36.41113、

44.24617、36.41113\}

$\mathrm{S}_{5}=\{44.72000 、$

44.72000、67.42912、

$44.72000 、 44.72000$ 、

67.42912、39.02720、

$18.83744 、 39.02720\}$

$\mathrm{S}_{5}=\{103.05832$ 、

122.41232、103.05832、 67.57920、44.37400、 $1 、 3$ 50.20000、43.35840、 $88.38272 、 98.32336\}$

$\mathrm{S}_{5}=\{103.38116$ 、

126.23296、103.38116、

$1 、 3 、 8$ 、 64.52448、31.18260、

31.18260、64.52448、

103.38116、103.38116\}

1、3、7、

9

2、 8

4、 6

1、2、4、 5

3、 6

7、 9

$S_{5}=\{130.72028 、$

61.20496、130.72028、 178.74128、146.39584、 83.28384、58.07444、

$130.64812 、 146.39584\}$

$\mathrm{S}_{5}=\{50.19680$ 、

45.24640、33.74720、

$33.74720 、 45.24640$ 、

$29.41184 、 15.82688$ 、

$29.41184 、 50.19680\}$

$\mathrm{S}_{5}=\{32.23040 、$

32.23040、41.08960、

$32.23040 、 32.23040$ 、

41.08960、32.23040、

1、3

5、 9

1、 9

2、 5

3、 4

6、 8

1、2、4、 5、7、8

3、 6 
Figures

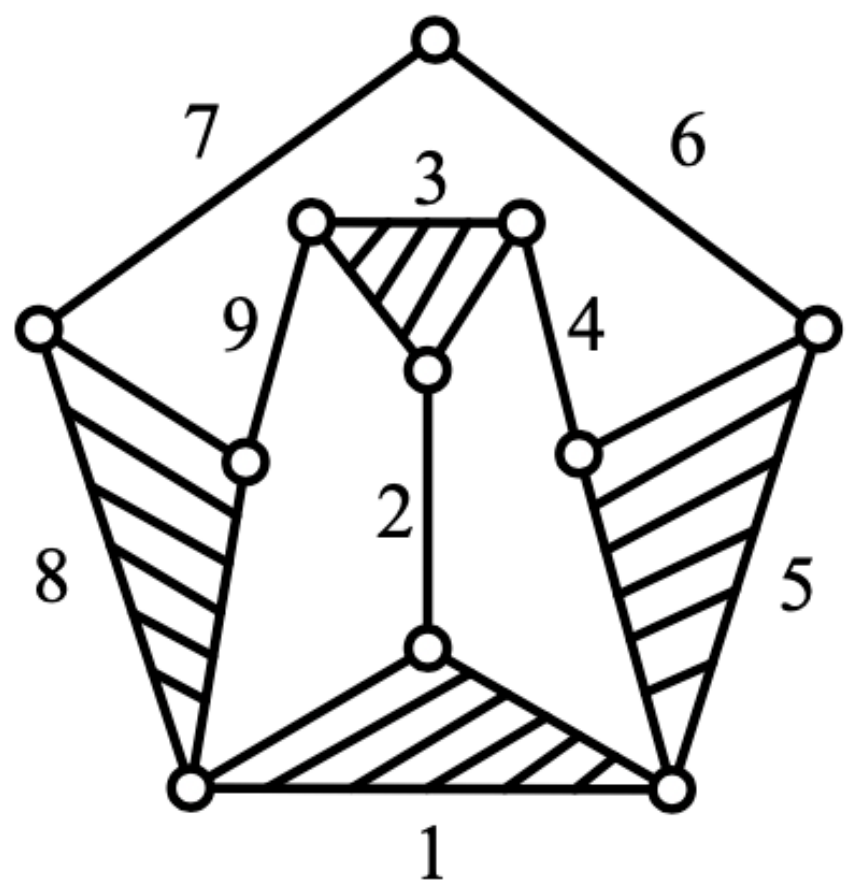

(a)

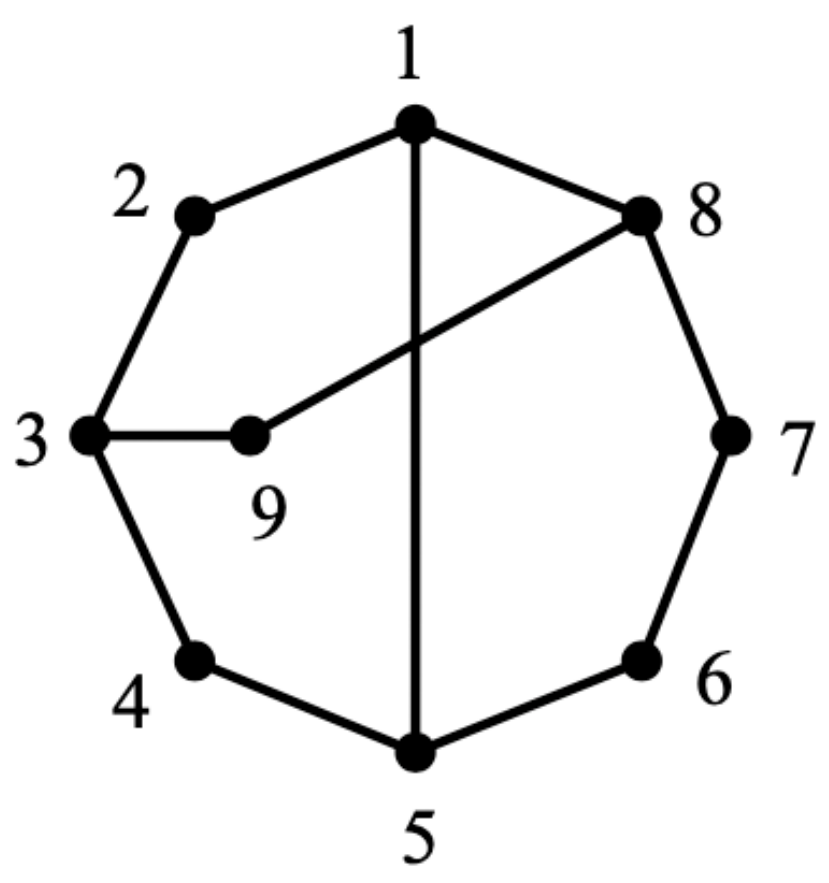

(b)

Figure 1

2-DOF KCs (a) and its corresponding topological diagram (b) 

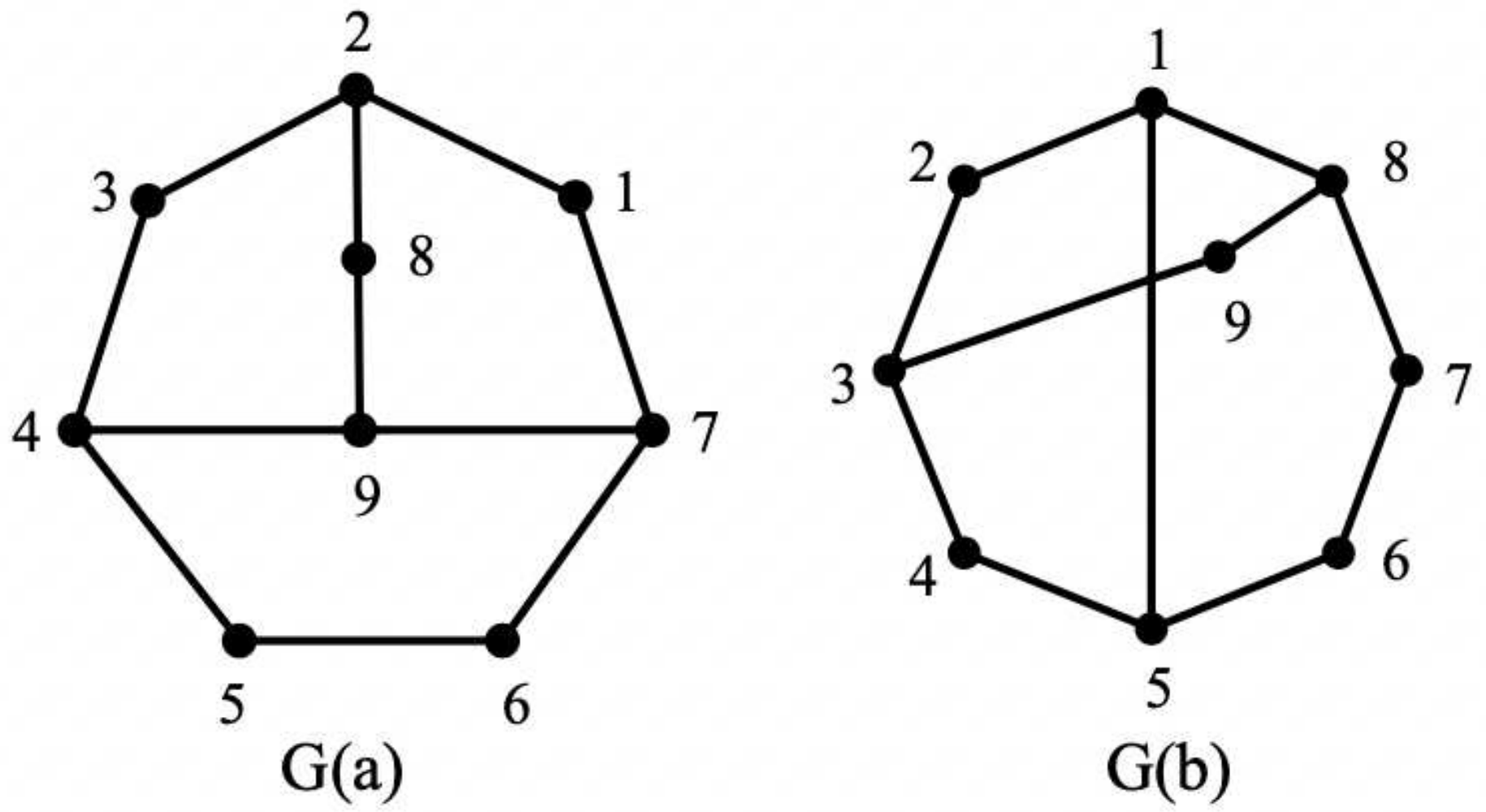

Figure 2

Isomorphic topological graph
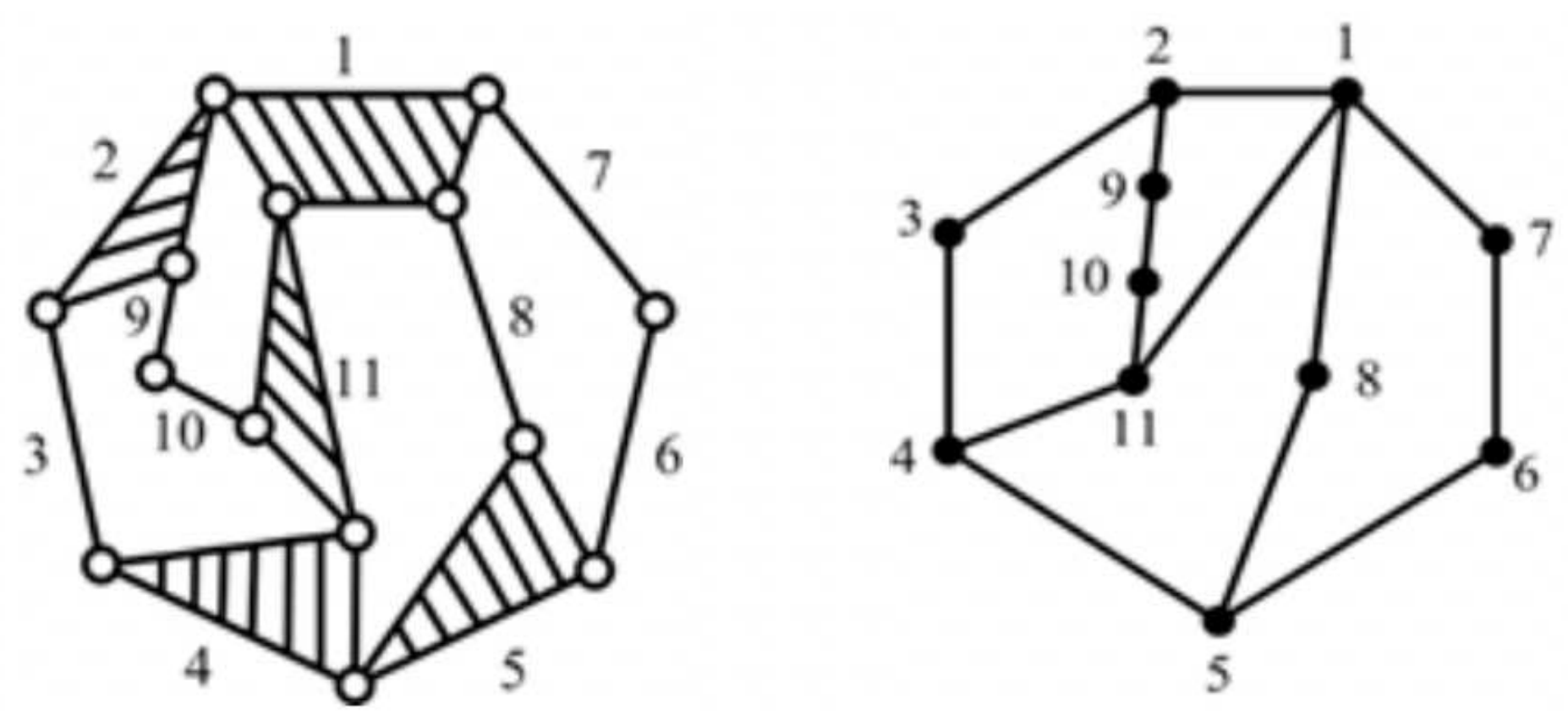

(a)

(b) 
Figure 3

11-link 2-DOF KCs (a) and its topological diagram (b)

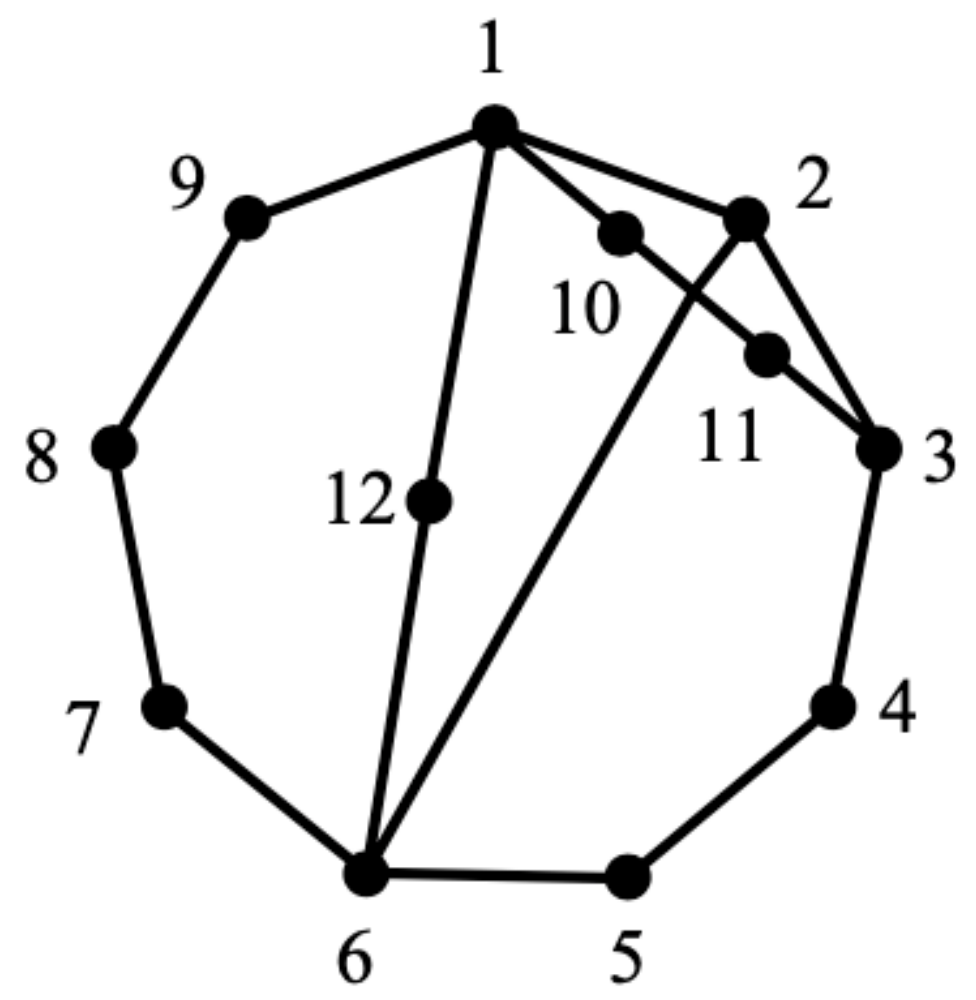

Figure 4

A topology with 12 vertices and 15 edges 


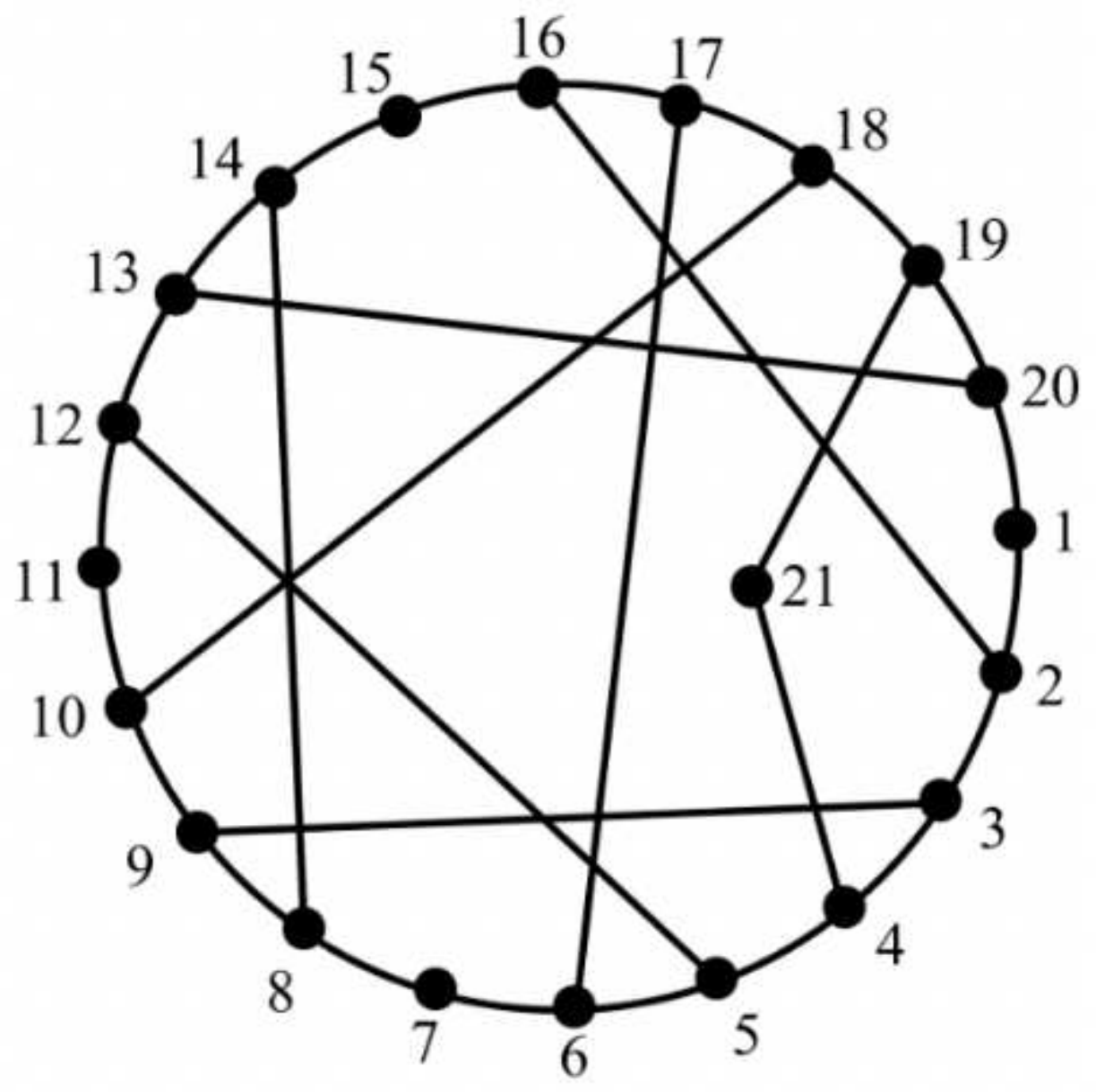

Figure 5

A topology with 21 vertices and 29 edges 


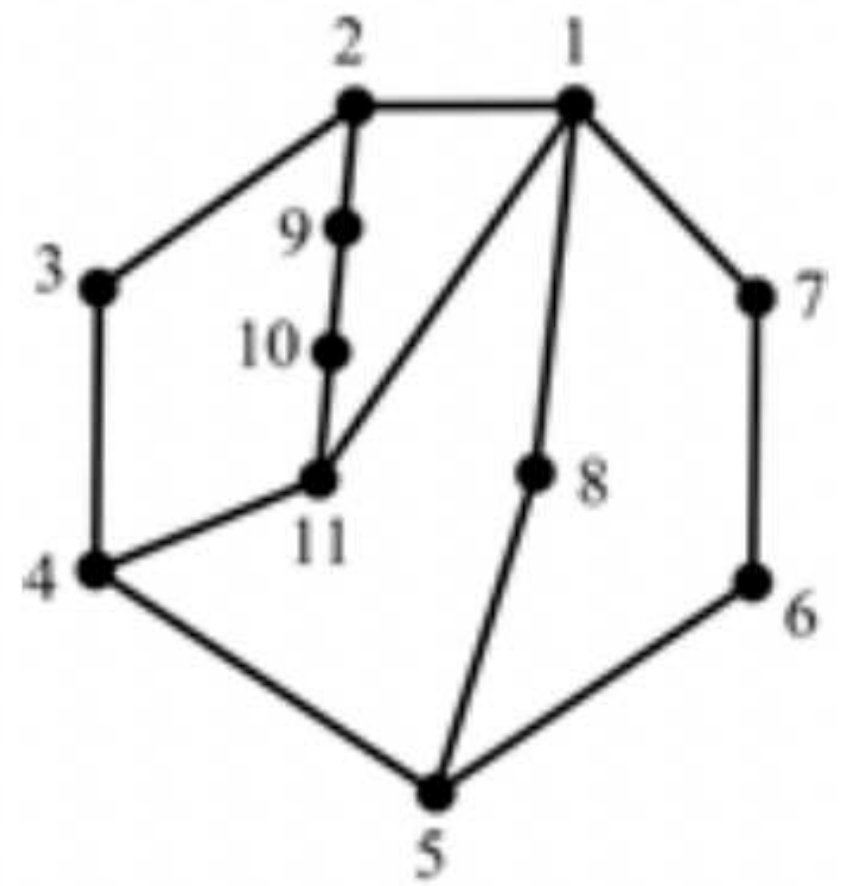

G(a)

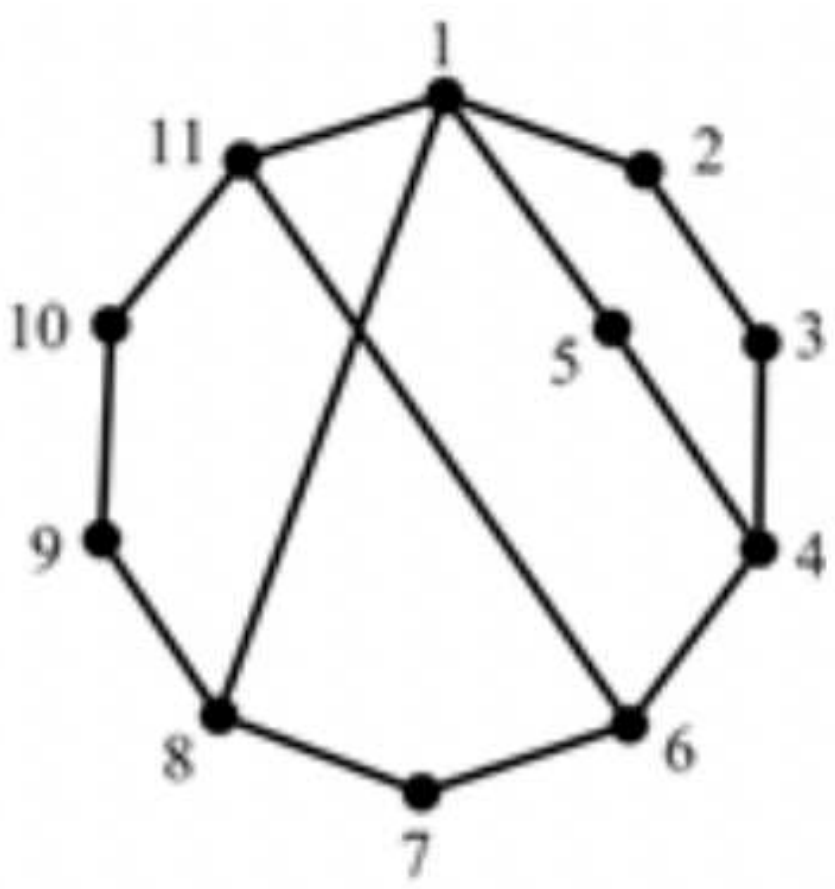

$\mathrm{G}(\mathrm{b})$

Figure 6

A topology with 11-link and 2-DOF

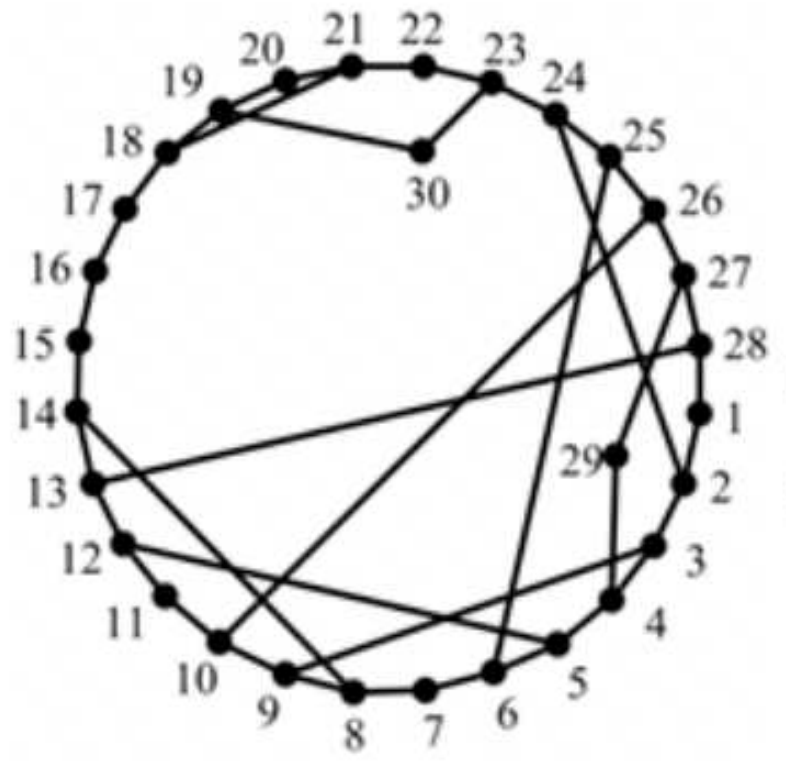

G(a)

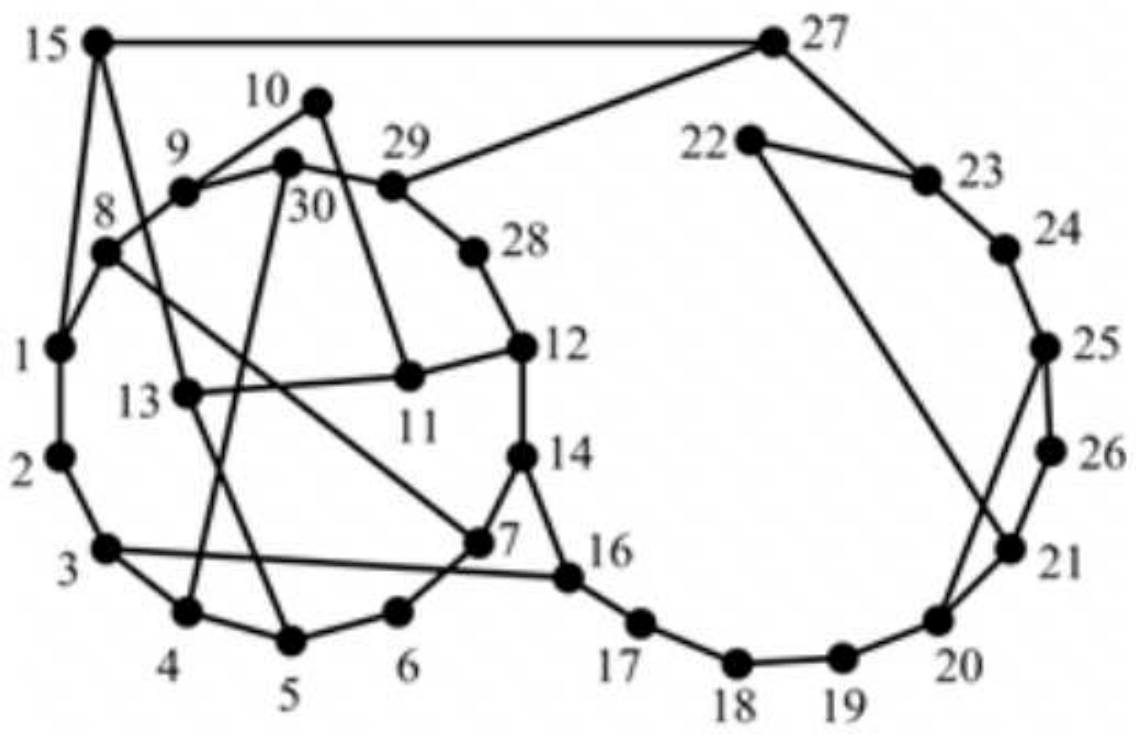

G(b)

Figure 7 
Topological diagrams with 30 vertices and 40 edges

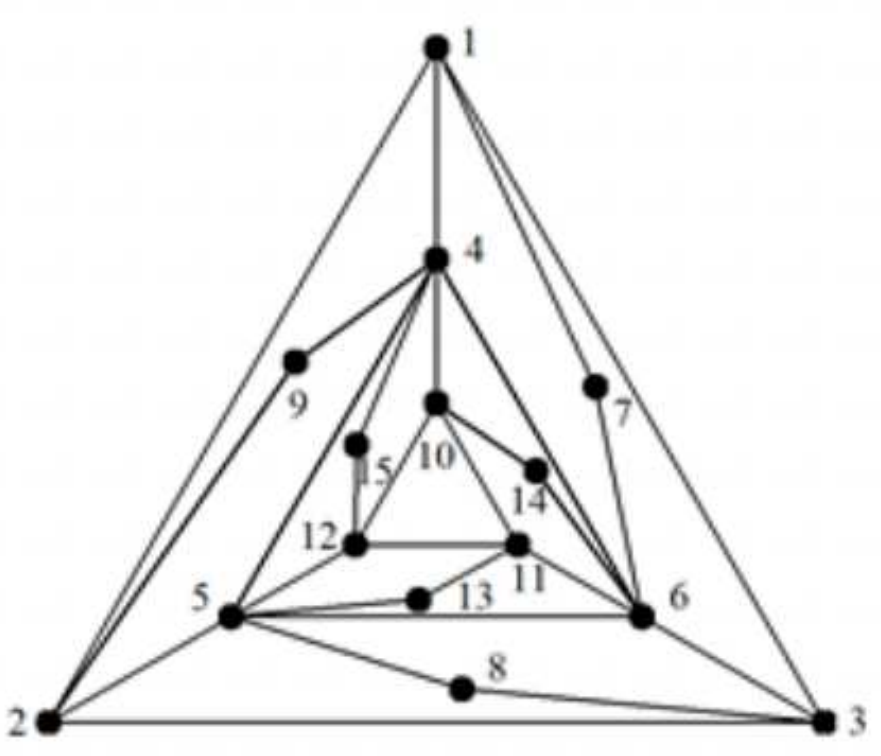

$\mathrm{G}(\mathrm{a})$

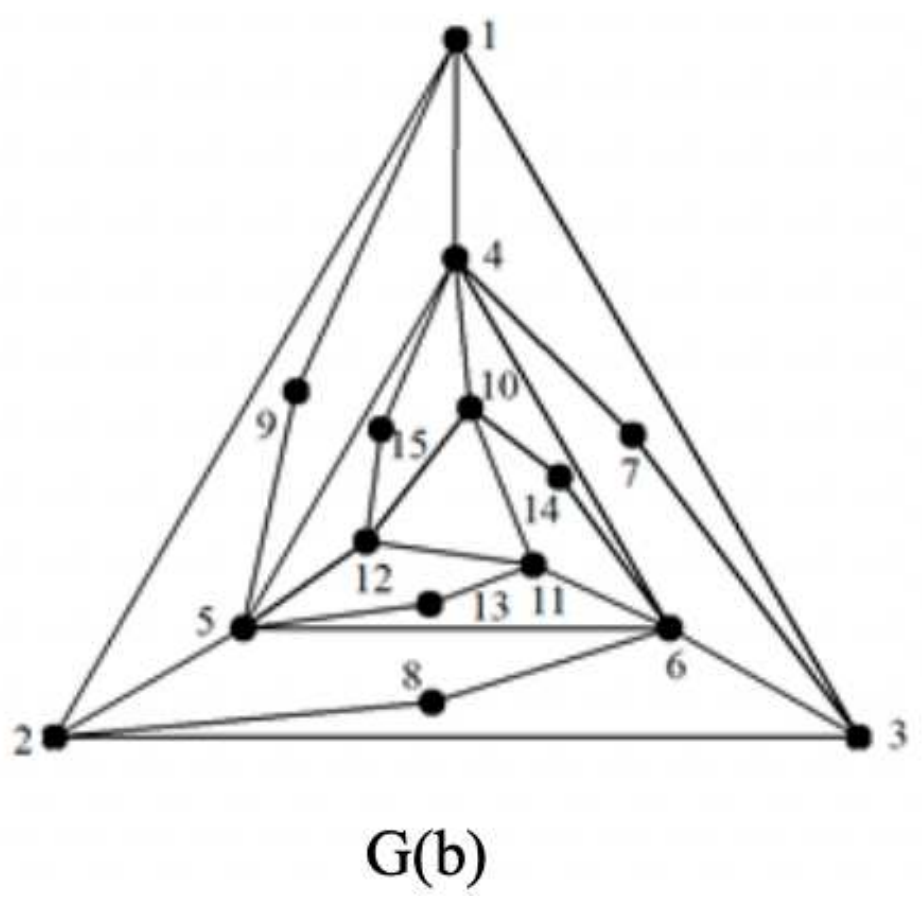

Figure 8

Topological diagrams with 15 vertices and 27 edges 